Homology, Homotopy and Applications, vol.9(2), 2007, pp.451-472

\title{
HOPF-HOCHSCHILD (CO)HOMOLOGY OF MODULE ALGEBRAS
}

\author{
ATABEY KAYGUN
}

(communicated by Claude Cibils)

\begin{abstract}
We define a version of Hochschild homology and cohomology suitable for a class of algebras admitting compatible actions of bialgebras, called module algebras. We show that this (co)homology, called Hopf-Hochschild (co)homology, can also be defined as a derived functor on the category of representations of an equivariant analogue of the enveloping algebra of a crossed product algebra. We investigate the relationship of our theory with Hopf cyclic cohomology and also prove Morita invariance of the Hopf-Hochschild (co)homology.
\end{abstract}

\section{Introduction}

Our goal in this paper is to define a version of Hochschild homology and cohomology suitable for a class of algebras admitting compatible actions of bialgebras, called "module algebras" (Definition 2.1). Our motivation lies in the following problem: for an algebra $A$ which admits a module structure over an arbitrary bialgebra $B$ compatible with its product structure, the Hochschild or the cyclic bicomplexes associated with this algebra need not be differential graded $B$-modules. The obstruction which prevents these complexes from being $B$-linear is trivial whenever the bialgebra $B$ is cocommutative, as in the case of group rings and universal enveloping algebras. Yet the same obstruction is far from being trivial if the underlying bialgebra is non-cocommutative. In this article, we will investigate how much of the Hochschild homology is retained after dividing this obstruction out. To this end, we will construct a new differential graded $B$-module $\mathrm{QCH}_{*}(A, B, V)$ (Proposition 2.12 and Definition 2.13) for a $B$-module algebra $A$ and a $B$-equivariant $A$-bimodule $V$ (Definition 2.2). We will define $H H_{*}^{\mathrm{Hopf}}(A, B, V)$, the Hopf-Hochschild homology of $A$ with coefficients in $V$, as the homology of the complex $\underset{B}{\otimes} \underset{\mathrm{QCH}}{\mathrm{CH}_{*}}(A, B, V)$. We would like to point out that the same strategy worked remarkably well in the case of cyclic cohomology of module coalgebras. In [13] we show that if we start with the cocyclic bicomplex of a module coalgebra twisted by a stable anti-YetterDrinfeld module, then dividing the analogous obstruction results in the Hopf cyclic

Received July 10, 2006, revised April 1, 2007; published on November 12, 2007.

2000 Mathematics Subject Classification: 16E40.

Key words and phrases: Hochschild cohomology, module algebra, Hopf algebra, bialgebra, Morita invariance.

Copyright (C) 2007, International Press. Permission to copy for private use granted. 
complex of [10], which was an extension of the Hopf cyclic cohomology of Connes and Moscovici [7].

In the context of cyclic (co)homology and $K$-theory, one of the most commonly used tools dealing with module algebras has been crossed product algebras. There is a large body of work dealing with algebras admitting actions of discrete groups and compact Lie groups, e.g. $[\mathbf{9}, \mathbf{1 1}, \mathbf{2}, \mathbf{5}, \mathbf{1 2}, \mathbf{8}, \mathbf{4}]$ and references therein, which utilizes this tool to its fullest extent. Also, there have been successful attempts in defining equivariant cyclic (co)homology and $K$-theory for module algebras over Hopf algebras $[\mathbf{3}, \mathbf{1}, \mathbf{1 8}]$ again by using crossed product algebras. Crossed product algebras enter in our picture in Corollary 4.4 where we show that Hopf-Hochschild homology can also be defined as a derived functor on the category of representations of an equivariant analogue of the enveloping algebra of a crossed product algebra (Definition 3.1).

The last result we prove is the Morita invariance of the Hopf-Hochschild homology and cohomology (Theorems 7.9 and 8.4). Our proof utilizes some additional tools from functor homology $[\mathbf{1 7}, \mathbf{1 9}]$. In doing so, we observe that the category of representations of a crossed product algebra is rather small for computing equivariant invariants. However, the shortcomings of this category can be overcome by using " $B$-categories" (Definition 6.1). We refer the reader to Remark 6.9 for a more detailed analysis on this subject.

Here is the plan of this paper: In Section 2 we give the basic definition of HopfHochschild complex of module algebras with coefficients in equivariant bimodules. We also point out the connections between Hopf-Hochschild homology and Hopf cyclic cohomology (Remark 2.15). In Section 3 we define Hopf-Hochschild cohomology of a $B$-module algebra and calculate it for lower dimensions. We also give a derived functor interpretation of the Hopf-Hochschild cohomology in terms of crossed product algebras. In Section 4 we extend the derived functor interpretation to Hopf-Hochschild homology. Sections 5 and 6 contain technical results needed toward proving Morita invariance of Hopf-Hochschild (co)homology in Section 7 in its full generality. In Section 8 we develop a generalized twisting method for coefficient bifunctors or bimodules by using Yetter-Drinfeld modules similar to the method of twisting developed in $[\mathbf{1 0}]$. In this last section, we also prove Morita invariance for twisted Hopf-Hochschild (co)homology.

Throughout this paper, we assume $k$ is an arbitrary field and $B$ is an associative/coassociative unital/counital bialgebra, or a Hopf algebra with an invertible antipode whenever it is necessary. All tensor products are taken over $k$ unless it is stated otherwise explicitly.

\section{Acknowledgements}

We would like to thank S. Witherspoon and the referee for their valuable comments and corrections. 
Homology, Homotopy and Applications, vol. 9(2), 2007

\section{Hopf-Hochschild homology}

Definition 2.1. An algebra $A$ is called a left $B$-module algebra if $A$ is a $B$-module and

$$
b\left(a_{1} a_{2}\right)=b_{(1)}\left(a_{1}\right) b_{(2)}\left(a_{2}\right)
$$

for any $b \in B$ and $a_{1}, a_{2} \in A$. If $A$ is unital, then we also assume $b\left(1_{A}\right)=\varepsilon(b) 1_{A}$ where $\varepsilon$ is the counit of $B$.

Recall that an $A$-bimodule is a left and right $A$-module which satisfies $a \triangleright(v \triangleleft$ $\left.a^{\prime}\right)=(a \triangleright v) \triangleleft a^{\prime}$ for any $a, a^{\prime} \in A$ and $v \in V$. Here we use $\triangleleft$ and $\triangleright$ to denote the right and left actions of $A$ on $V$ respectively.

Definition 2.2. Let $A$ be a $B$-module algebra. An $A$-module $V$ is called a $B$ equivariant $A$-bimodule if $V$ is both an $A$-bimodule and $B$-module and one also has

$$
b\left(a^{\prime} \triangleright v \triangleleft a\right)=\left(b_{(1)} a^{\prime}\right) \triangleright\left(b_{(2)} v\right) \triangleleft\left(b_{(3)} a\right)
$$

for any $a, a^{\prime} \in A$ and $b \in B$.

Example 2.3. Let $B=k[G]$ be the group algebra of a discrete group $G$. Then an algebra $A$ is a $k[G]$-module algebra if and only if $A$ admits an action of $G$ by algebra automorphisms.

Example 2.4. Let $B=U(\mathfrak{g})$ be the universal enveloping algebra of Lie algebra $\mathfrak{g}$. Then an algebra $A$ is a $U(\mathfrak{g})$-module algebra if and only if $A$ admits an action of $\mathfrak{g}$ by derivations.

Example 2.5. Let $B$ be an arbitrary Hopf algebra and $A$ be an algebra in the category of $B$-bimodules, and let $V$ be a left $A$-module in the category of left $B$-modules, where in both cases we use tensor product over $B$ as our monoidal product. These conditions require that we have

$$
\begin{aligned}
& (b a) a^{\prime}=b\left(a a^{\prime}\right), \quad a\left(a^{\prime} b\right)=\left(a a^{\prime}\right) b, \quad(a b) a^{\prime}=a\left(b a^{\prime}\right), \\
& (b a) v=b \triangleright(a v), \quad(a b) v=a(b \triangleright v)
\end{aligned}
$$

for any $a, a^{\prime} \in A, b \in B$ and $v \in V$. If $A$ is a unital algebra, then $A$ necessarily admits a morphism of algebras $\gamma: B \rightarrow A$ defined by $\gamma(b):=b\left(1_{A}\right)$. Then we have $b a b^{\prime}:=\gamma(b) a \gamma\left(b^{\prime}\right)$ and $b \triangleright v:=\gamma(b) v$ for any $b, b^{\prime} \in B, a \in A$ and $v \in V$. In this setup, there is a natural action of $B$ on $A$ called the adjoint action which makes $A$ into a $B$-module algebra and $V$ into a $B$-equivariant $A$-module. The adjoint action is defined as

$$
a d_{b}(a)=b_{(1)} a S\left(b_{(2)}\right)
$$

for any $b \in B$ and $a \in A$. One can easily see that

$$
a d_{b}\left(a_{1} a_{2}\right)=b_{(1)}\left(a_{1} a_{2}\right) S\left(b_{(2)}\right)=b_{(1)} a_{1} S\left(b_{(2)}\right) b_{(3)} a_{2} S\left(b_{(4)}\right)=a d_{b_{(1)}}\left(a_{1}\right) a d_{b_{(2)}}\left(a_{2}\right)
$$

for any $b \in B$ and $a_{1}, a_{2} \in A$. Similarly,

$$
b(a \triangleright v)=b_{(1)} a S\left(b_{(2)}\right) \triangleright b_{(3)} v=a d_{b_{(1)}}(a) \triangleright\left(b_{(2)} v\right)
$$

for any $a \in A, b \in B$ and $v \in V$. 
Example 2.6. Consider the Hopf algebra $\mathcal{O}$ generated (as an algebra) by symbols $D_{n}, \alpha$ and $\alpha^{-1}$ where $n \in \mathbb{Z}$, and we have the relations

$$
\alpha D_{n} \alpha^{-1}=D_{n+1} \quad \text { for any } n \in \mathbb{Z} .
$$

We also let

$$
\Delta\left(\alpha^{\ell}\right)=\alpha^{\ell} \otimes \alpha^{\ell} \text { for } \ell \in \mathbb{Z} \quad \text { and } \quad \Delta\left(D_{n}\right)=\left(\alpha \otimes D_{n}\right)+\left(D_{n} \otimes 1\right) \text { for } n \in \mathbb{Z} .
$$

This data is enough to define a Hopf algebra structure on $\mathcal{O}$. One can easily observe that an algebra $A$ is an $\mathcal{O}$-module algebra if and only if $A$ admits an algebra automorphism $\alpha$ and an $\alpha$-derivation $\delta$.

Example 2.7. Let $\mathcal{H}_{1}$ be the Hopf algebra generated by the symbols $X, Y$ and $\delta_{n}$, where $n \geqslant 1$ is an integer. We impose the following relations

$$
[Y, X]=X, \quad\left[Y, \delta_{1}\right]=\delta_{1}, \quad\left[X, \delta_{n}\right]=\delta_{n+1}, \quad\left[\delta_{n+1}, \delta_{n}\right]=0,
$$

for all possible $n \geqslant 1$, where we use $[a, b]:=a b-b a$ for arbitrary elements $a, b \in \mathcal{H}_{1}$. We also let

$$
\begin{aligned}
& \Delta(Y)=(Y \otimes 1)+(1 \otimes Y), \\
& \Delta\left(\delta_{1}\right)=\left(\delta_{1} \otimes 1\right)+\left(1 \otimes \delta_{1}\right), \\
& \Delta(X)=(X \otimes 1)+(1 \otimes X)+\left(\delta_{1} \otimes Y\right) .
\end{aligned}
$$

One can define $\Delta\left(\delta_{n}\right)$ recursively by using the relation $\delta_{n+1}=\left[X, \delta_{n}\right]$ and the fact that $\Delta$ is a morphism of algebras. This data is enough to define a Hopf algebra structure on $\mathcal{H}_{1}$. Although our presentation is slightly different, the Hopf algebra $\mathcal{H}_{1}$ is the the same as Connes-Moscovici Hopf algebra defined in [7].

One can define an $\mathcal{H}_{1}$-module algebra structures on the algebra of meromorphic functions on $\mathbb{C}$ by letting

$$
\begin{aligned}
\delta_{1}(f(z)) & :=f^{\prime}(z) \delta_{1}(z), \\
Y(f(z)) & :=f^{\prime}(z) Y(z), \\
X(f(z)) & :=\frac{1}{2} \delta_{1}(z) Y(z) f^{\prime \prime}(z)+X(z) f^{\prime}(z),
\end{aligned}
$$

where we use $\delta_{1}(z), Y(z)$ and $X(z)$ to denote the action of each generator on the function $f(z)=z$. By using the relations in $\mathcal{H}_{1}$, one can show that the choices of $Y(z)$ and $X(z)$ depend on the choice of $\delta_{1}(z)$ as

$$
Y(z)=-\delta_{1}(z) \int \frac{1}{\delta_{1}(z)} d z \quad \text { and } \quad X(z)=\frac{1}{2} Y(z) \frac{d}{d z} \delta_{1}(z)+c \delta_{1}(z)
$$

where $c$ is an arbitrary constant, provided $Y(z)$ is meromorphic. If we choose $\delta_{1}(z)$ in the algebra of polynomials $\mathbb{C}[z]$ and if $Y(z)$ happens to be a polynomial too, then the same data determines an $\mathcal{H}_{1}$-module algebra structure on $\mathbb{C}[z]$ as well. For example, if we let $\delta_{1}(z)=z^{2}$, then $Y(z)=a z^{2}+z$ and $X(z)=a z^{3}+b z^{2}$ for some constants $a, b \in \mathbb{C}$, and we obtain an $\mathcal{H}_{1}$-module algebra structure on the algebra of polynomials $\mathbb{C}[z]$. 
Definition 2.8. Given an algebra $A$ and a $A$-bimodule $V$, we will use the notation $\mathrm{CH}_{*}(A, V)$ to denote graded module $\bigoplus_{n \geqslant 0} V \otimes A^{\otimes n}$ with structure morphisms

$$
\partial_{j}\left(v \otimes a_{1} \otimes \cdots \otimes a_{n}\right),= \begin{cases}\left(v \triangleleft a_{1} \otimes a_{2} \otimes \cdots \otimes a_{n}\right), & \text { if } j=0 ; \\ \left(v \otimes \cdots \otimes a_{j} a_{j+1} \otimes \cdots\right), & \text { if } 1 \leqslant j<n-1 ; \\ \left(a_{n} \triangleright v \otimes a_{1} \otimes \cdots \otimes a_{n-1}\right), & \text { if } j=n,\end{cases}
$$

which makes $\mathrm{CH}_{*}(A, V)$ into a pre-simplicial module. The differential graded module with the differentials

$$
d_{n}^{\mathrm{CH}}=\sum_{j=0}^{n}(-1)^{j} \partial_{j}
$$

corresponding to this pre-simplicial module is also denoted by $\mathrm{CH}_{*}(A, V)$, and is called the Hochschild complex of $A$ with coefficients in the $A$-bimodule $V$.

From this point on, we will assume $A$ is a $B$-module algebra and $V$ is a $B$ equivariant $A$-bimodule unless it is stated otherwise explicitly.

Remark 2.9. $B$ as an algebra acts on $\mathrm{CH}_{*}(A, V)$ diagonally as

$$
L_{b}\left(v \otimes a_{1} \otimes \cdots \otimes a_{n}\right)=b_{(1)}(v) \otimes b_{(2)}\left(a_{1}\right) \otimes \cdots \otimes b_{(n+1)}\left(a_{n}\right),
$$

which makes $\mathrm{CH}_{*}(A, V)$ into a graded $B$-module but NOT a differential graded $B$-module since, in general,

$$
\partial_{n} L_{b}\left(v \otimes a_{1} \otimes \cdots \otimes a_{n}\right)=b_{(n+1)}\left(a_{n}\right) \triangleright b_{(1)}(v) \otimes b_{(2)}\left(a_{1}\right) \otimes \cdots \otimes b_{(n)}\left(a_{n-1}\right)
$$

need not be the same as

$$
L_{b} \partial_{n}\left(v \otimes a_{1} \otimes \cdots \otimes a_{n}\right)=b_{(1)}\left(a_{n}\right) \triangleright b_{(2)}(v) \otimes b_{(3)}\left(a_{1}\right) \otimes \cdots \otimes b_{(n+1)}\left(a_{n-1}\right)
$$

for any $n \geqslant 0, a_{1} \otimes \cdots \otimes a_{n} \otimes v \in \mathrm{CH}_{n}(A, V)$ and $b \in B$. One interesting case when $\partial_{n} L_{b}=L_{b} \partial_{n}$ is when $B$ is cocommutative, i.e. $b_{(1)} \otimes b_{(2)}=b_{(2)} \otimes b_{(1)}$ for any $b \in B$. The best-known examples of cocommutative bialgebras are group rings of discrete groups and universal enveloping algebras of Lie algebras. This means that the graded $B$-module structure on the ordinary Hochschild complex $\mathrm{CH}_{*}(A, V)$ extends to a differential graded $B$-module structure on $\mathrm{CH}_{*}(A, V)$ when $B$ is cocommutative. The obstruction which prevents $\mathrm{CH}_{*}(A, V)$ from being a differential graded $B$ module is the subcomplex generated by images of the the commutators $\left[L_{b}, d_{*}^{\mathrm{CH}}\right]=$ $(-1)^{n}\left[L_{b}, \partial_{n}\right]$ where $L_{b}$ is the $k$-linear endomorphism of $\mathrm{CH}_{*}(A, V)$ coming from the diagonal action of $b \in B$ on $\mathrm{CH}_{*}(A, V)$. Now one can ask the following question: what happens if we force these differential graded $k$-modules to become differential graded $B$-modules by dividing out this obstruction? This is what we are going to do with Definition 2.11 and Proposition 2.12 for the ordinary Hochschild complex, and then we will investigate homological consequences of this operation.

Let $\left(\mathcal{C}_{*}, d_{*}^{\mathcal{C}}\right)$ be a differential graded $k$-module and let $n \in \mathbb{N}$. Then we define $\mathcal{C}_{*}[+n]$, the $n$-fold suspension of $\mathcal{C}_{*}$, as the differential graded $k$-module $\mathcal{C}_{m}[+n]=$ $\mathcal{C}_{m+n}$ with differentials $d_{m}^{\mathcal{C}}[+n]=d_{m+n}^{\mathcal{C}}$ for any $m \in \mathbb{Z}$. One can similarly define $\mathcal{C}_{*}[-n]$ for any $n \in \mathbb{N}$. Note that $H_{m \pm n}\left(\mathcal{C}_{*}[ \pm n]\right)=H_{m}\left(\mathcal{C}_{*}\right)$. 
Lemma 2.10. For any $b \in B$, there is a morphism of differential graded $k$-modules of the form

$$
\mathrm{CH}_{*}(A, V)[+1] \stackrel{\left[L_{b}, \partial_{*+1}\right]}{\longrightarrow} \mathrm{CH}_{*}(A, V) .
$$

Moreover, $\left[L_{b}, \partial_{*+1}\right]$ is null-homotopic for any $b \in B$.

Proof. For any $b \in B$ and $v \otimes \mathbf{a} \in \mathrm{CH}_{n}(A, V)[+1]$, we consider

$$
\begin{aligned}
\partial_{j}\left[L_{b}, \partial_{n+1}\right] & (v \otimes \mathbf{a})=-\left[L_{b}, \partial_{j}\right] \partial_{n+1}(v \otimes \mathbf{a}),+\left[L_{b}, \partial_{j} \partial_{n+1}\right](v \otimes \mathbf{a}), \\
& = \begin{cases}{\left[L_{b}, \partial_{n} \partial_{j}\right](v \otimes \mathbf{a}),} & \text { if } 0 \leqslant j \leqslant n-1 ; \\
-\left[L_{b}, \partial_{n}\right] \partial_{n+1}(v \otimes \mathbf{a})+\left[L_{b}, \partial_{n} \partial_{n}\right](v \otimes \mathbf{a}), & \text { if }=n ;\end{cases} \\
& = \begin{cases}{\left[L_{b}, \partial_{n}\right] \partial_{j}(v \otimes \mathbf{a}),} & \text { if } 0 \leqslant j \leqslant n-1 ; \\
-\left[L_{b}, \partial_{n}\right] \partial_{n+1}(v \otimes \mathbf{a})+\left[L_{b}, \partial_{n}\right] \partial_{n}(v \otimes \mathbf{a}), & \text { if } j=n,\end{cases}
\end{aligned}
$$

by using the fact that $\left[L_{b}, \partial_{j}\right](v \otimes \mathbf{a})=0$ for any $n \geqslant 0$ and $0 \leqslant j \leqslant n-1$. This immediately implies

$$
\begin{aligned}
d_{n}^{\mathrm{CH}}\left[L_{b}, \partial_{n+1}\right] & =\sum_{j=0}^{n-1}\left[L_{b}, \partial_{n}\right] \partial_{j}+(-1)^{n}\left[L_{b}, \partial_{n}\right] \partial_{n}+(-1)^{n+1}\left[L_{b}, \partial_{n}\right] \partial_{n+1} \\
& =\left[L_{b}, \partial_{n}\right] d_{n+1}^{\mathrm{CH}}=\left[L_{b}, \partial_{n}\right] d_{n}^{\mathrm{CH}[+1]} .
\end{aligned}
$$

The null-homotopy $\mathrm{CH}_{n}(A, V)[+1] \stackrel{s_{n}}{\longrightarrow} \mathrm{CH}_{n}(A, V)[+1]$ is given by $s_{n}=(-1)^{n} L_{b}$ for any $n \geqslant 0$.

Definition 2.11. We define a graded $B$-submodule of $\mathrm{CH}_{*}(A, V)$ as

$$
J_{*}(A, B, V)=\sum_{b \in B} i m\left(\left[L_{b}, \partial_{*+1}\right]\right) .
$$

Proposition 2.12. We define a new graded $B$-module $\mathrm{QCH}_{*}(A, B, V)$ as the quotient graded B-module

$$
\mathrm{QCH}_{*}(A, B, V):=\mathrm{CH}_{*}(A, V) / J_{*}(A, B, V) .
$$

Then $\mathrm{QCH}_{*}(A, B, V)$ is also a differential graded $B$-module.

Proof. Since each $i m\left(\left[L_{b}, \partial_{*+1}\right]\right)$ is a differential graded $k$-submodule of $\mathrm{CH}_{*}(A, V)$, the submodule $J_{*}(A, B, V)$ is also a differential graded $k$-submodule of $\mathrm{CH}_{*}(A, V)$. This fact implies that $\mathrm{QCH}_{*}(A, B, V)$ is also a differential graded $k$-module. Moreover, $J_{*}(A, B, V)$ is a graded $B$-submodule of $\mathrm{CH}_{*}(A, V)$ since

$$
L_{x}\left[L_{b}, \partial_{n+1}\right](v \otimes \mathbf{a})=-\left[L_{x}, \partial_{n+1}\right] L_{b}(v \otimes \mathbf{a})+\left[L_{x b}, \partial_{n+1}\right](v \otimes \mathbf{a})
$$

for any $x, b \in B, n \geqslant 0$ and $(v \otimes \mathbf{a})$ in $\mathrm{CH}_{n}(A, V)[+1]$. For $\mathrm{QCH}_{*}(A, B, V)$ to be a differential graded $B$-module, we must show that $\left[L_{b}, d_{*}^{\mathrm{CH}}\right] \equiv 0$ on $\mathrm{QCH}_{*}(A, B, V)$ for any $b \in B$. This is equivalent to saying that

$$
\left[L_{b}, d_{n}^{\mathrm{CH}}\right](v \otimes \mathbf{a})=(-1)^{n}\left[L_{b}, \partial_{n}\right](v \otimes \mathbf{a})
$$

must be in $J_{n-1}(B, B, V)$ for any $(v \otimes \mathbf{a})$ in $\mathrm{CH}_{n}(A, V)$ and for any $b \in B$ is in $J_{n-1}(A, B, V)$, which is true by definition. 
Definition 2.13. Assume $B$ is a bialgebra. For a $B$-module algebra $A$ and a $B$ equivariant $A$-module $V$, we define Hopf-Hochschild homology of $A$ with coefficients in $V$ as the homology of the differential graded $k$-module ${ }_{B} \mathrm{QCH}_{*}(A, B, V)$ which is defined as $k \underset{B}{\otimes} \mathrm{QCH}_{*}(A, B, V)$. In other words,

$$
H H_{n}^{\mathrm{Hopf}}(A, V):=H_{n}\left({ }_{B} \mathrm{QCH}_{*}(A, B, V)\right)
$$

for any $n \geqslant 0$.

Remark 2.14. Observe that if $B$ is cocommutative, then the differential graded $k$ module $\mathrm{QCH}_{*}(A, B, V)$ is equal to the ordinary Hochschild complex $\mathrm{CH}_{*}(A, V)$. In the case when $B$ is both cocommutative and semi-simple (such as $B=k[G]$ where $G$ is a finite group and $\operatorname{char}(k)$ does not divide $|G|)$, then one has an isomorphism of the form $H H_{*}^{\text {Hopf }}(A, V) \cong k \underset{B}{\otimes} H H_{*}(A, V)$. For example, if $B=k$, then the HopfHochschild homology is the same as the ordinary Hochschild homology.

Remark 2.15. Assume $A$ is an associative, but not necessarily unital $k$-algebra. Apart from the ordinary Hochschild complex of $A$, there are several other different differential graded $k$-modules one can associate with $A$ :

1. Connes' complex $\operatorname{CC}_{*}^{\lambda}(A)$ which is defined as the cyclic coinvariants of the ordinary Hochschild complex,

2. the positive, negative and periodic cyclic bicomplexes $\mathrm{CC}_{*}(A), \mathrm{CN}_{*}(A)$ and $\mathrm{CC}_{*}^{\mathrm{per}}(A)$,

3. the mixed complex $\mathrm{CM}_{*}(A)$, which is also referred as "the $(b, B)$-complex" which also has two other variations: the negative mixed complex $\operatorname{CM}_{*}^{-}(A)$ and the periodic mixed complex $\mathrm{CM}_{*}^{\text {per }}(A)$.

As before, the cyclic bicomplexes and the mixed complexes are graded $B$-modules but are not necessarily differential graded $B$-modules. The obstruction to extending the graded $B$-module structure to a differential graded $B$-module structure stems from the fact that the cyclic permutations and the diagonal $B$-action on the tensor powers of $A$ do not necessarily commute. We will investigate the consequences of the operation of dividing out this obstruction on the cyclic complexes we mentioned above in a different paper [14] in a more general set-up where the complexes are twisted by some coefficient module. We would like to point out that the obstruction which prevents the cyclic bicomplex from being a differential graded $B$-module is a larger differential graded submodule in the sense that the "Hochschild subcomplex" or the " $b$-subcomplex" of the Hopf cyclic bicomplex is a quotient of the Hopf-Hochschild complex we define here.

\section{Hopf-Hochschild cohomology}

In this section, we assume $A$ is an associative, not necessarily commutative, unital $B$-module algebra unless otherwise explicitly stated. 
Definition 3.1. We define $A^{e} \rtimes B$ as $A \otimes A \otimes B$ with the multiplication

$$
\left(a_{1} \otimes a_{1}^{\prime} \otimes b\right)\left(a_{2} \otimes a_{2}^{\prime} \otimes b^{\prime}\right)=a b_{(1)}\left(a_{2}\right) \otimes b_{(3)}\left(a_{2}^{\prime}\right) a_{1}^{\prime} \otimes b_{(2)} b^{\prime}
$$

for any $\left(a_{1} \otimes a_{1}^{\prime} \otimes b\right)$ and $\left(a_{2} \otimes a_{2}^{\prime} \otimes b^{\prime}\right)$ in $A^{e} \rtimes B$.

Lemma 3.2. $A^{e} \rtimes B$ is an unital associative algebra.

Proof. For associativity, one must consider

$$
\begin{aligned}
\left(\left(a_{1} \otimes a_{1}^{\prime} \otimes b\right)\right. & \left.\left(a_{2} \otimes a_{2}^{\prime} \otimes b^{\prime}\right)\right)\left(a_{3} \otimes a_{3}^{\prime} \otimes b^{\prime \prime}\right) \\
& =\left(a_{1} b_{(1)}\left(a_{2}\right) \otimes b_{(3)}\left(a_{2}^{\prime}\right) a_{1}^{\prime} \otimes b_{(2)} b^{\prime}\right)\left(a_{3} \otimes a_{3}^{\prime} \otimes b^{\prime \prime}\right) \\
& =a_{1} b_{(1)}\left(a_{2}\right) b_{(2)} b_{(1)}^{\prime}\left(a_{3}\right) \otimes b_{(4)} b_{(3)}^{\prime}\left(a_{3}^{\prime}\right) b_{(5)}\left(a_{2}^{\prime}\right) a_{1}^{\prime} \otimes b_{(3)} b_{(2)}^{\prime} b^{\prime \prime} \\
& =a_{1} b_{(1)}\left(a_{2} b_{(1)}^{\prime}\left(a_{3}\right)\right) \otimes b_{(3)}\left(b_{(3)}^{\prime}\left(a_{3}^{\prime}\right) a_{2}^{\prime}\right) a_{1}^{\prime} \otimes b_{(2)} b_{(2)}^{\prime} b^{\prime \prime} \\
& =\left(a_{1} \otimes a_{1}^{\prime} \otimes b\right)\left(\left(a_{2} \otimes a_{2}^{\prime} \otimes b^{\prime}\right)\left(a_{3} \otimes a_{3}^{\prime} \otimes b^{\prime \prime}\right)\right)
\end{aligned}
$$

for any $a_{i}, a_{i}^{\prime} \in A$ for $i=1,2,3$ and $b, b^{\prime}, b^{\prime \prime} \in B$.

Lemma 3.3. $B$-equivariant $A$-bimodule structures on $V$ are in bijective correspondence with $A^{e} \rtimes B$-module structures on $V$.

Proof. $V$ is a $B$-equivariant $A$-bimodule if and only if one has

$$
\begin{aligned}
\left(a_{1} \otimes a_{2} \otimes b\right)\left(\left(a_{1}^{\prime} \otimes a_{2}^{\prime} \otimes b^{\prime}\right) v\right) & =a_{1} \triangleright b\left(a_{1}^{\prime} \triangleright b^{\prime} v \triangleleft a_{2}^{\prime}\right) \triangleleft a_{2} \\
& =a_{1}\left(b_{(1)} a_{1}^{\prime}\right) \triangleright\left(b_{(2)} b^{\prime} v\right) \triangleleft\left(b_{(3)} a_{2}^{\prime}\right) a_{2} \\
& =\left(\left(a_{1} \otimes a_{2} \otimes b\right)\left(a_{1}^{\prime} \otimes a_{2}^{\prime} \otimes b^{\prime}\right)\right) v
\end{aligned}
$$

for any $\left(a_{1} \otimes a_{2} \otimes b\right)$ and $\left(a_{1}^{\prime} \otimes a_{2}^{\prime} \otimes b^{\prime}\right)$ in $A^{e} \rtimes B$ and $v \in V$; i.e., $V$ is a $A^{e} \rtimes B$ module.

Definition 3.4. Define a differential graded $k$-module $\mathrm{CB}_{*}(A)$ by letting $\mathrm{CB}_{n}(A)=$ $A^{\otimes n+2}$ for any $n \geqslant 0$. Then we define pre-simplicial structure morphisms

$$
\partial_{j}\left(a_{0} \otimes \cdots \otimes a_{n+1}\right)=\left(a_{0} \otimes \cdots \otimes a_{j} a_{j+1} \otimes \cdots \otimes a_{n+1}\right)
$$

for any $n \geqslant 1$ and $0 \leqslant j \leqslant n$ and then define the differentials as $d_{n}^{\mathrm{CB}}=\sum_{j=0}^{n}(-1)^{j} \partial_{j}$ for any $n \geqslant 1$. One can see that $\mathrm{CB}_{*}(A)$ is a graded $A$-bimodule where $A$-actions are defined as

$$
a\left(a_{0} \otimes \cdots \otimes a_{n+1}\right)=a a_{0} \otimes \cdots \otimes a_{n+1}, \quad\left(a_{0} \otimes \cdots \otimes a_{n+1}\right) a=a_{0} \otimes \cdots \otimes a_{n+1} a
$$

for any $a \in A$ and $\left(a_{0} \otimes \cdots \otimes a_{n+1}\right)$ in $\mathrm{CB}_{n}(A)$. There is also a left graded $B$-module structure defined for $b \in B$ and $\left(a_{0} \otimes \cdots \otimes a_{n+1}\right)$ in $\mathrm{CB}_{n}(A)$ as

$$
b\left(a_{0} \otimes \cdots \otimes a_{n+1}\right)=b_{(1)} a_{0} \otimes \cdots \otimes b_{(n+2)} a_{n+1} .
$$

This makes $\mathrm{CB}_{*}(A)$ into a left $A^{e} \rtimes B$-module since one has

$$
\begin{aligned}
b\left(a \otimes a^{\prime}\right)\left(a_{0} \otimes \cdots \otimes a_{n+1}\right) & =b_{(1)}\left(a a_{0}\right) \otimes b_{(2)} a_{1} \otimes \cdots \otimes b_{(n+1)} a_{n} \otimes b_{(n+2)}\left(a_{n+1} a^{\prime}\right) \\
& =b_{(1)}(a) b_{(2)}\left(a_{0}\right) \otimes b_{(3)} a_{1} \otimes \cdots \otimes b_{(n+3)}\left(a_{n+1}\right) b_{(n+4)}\left(a^{\prime}\right) \\
& =\left(b_{(1)}(a) \otimes b_{(3)}\left(a^{\prime}\right)\right)\left(b_{(2)}\left(a_{0} \otimes \cdots \otimes a_{n+1}\right)\right),
\end{aligned}
$$

$b \in B,\left(a \otimes a^{\prime}\right)$ in $A^{e}$ and $\left(a_{0} \otimes \cdots \otimes a_{n+1}\right)$ in $\mathrm{CB}_{n}(A)$. 
The proof of the following lemma is routine.

Lemma 3.5. $\mathrm{CB}_{*}(A)$ is a differential graded $A^{e} \rtimes B$-module.

Definition 3.6. Let $A$ be a $B$-module algebra and let $V$ be an $B$-equivariant $A$ bimodule. Define the Hopf-Hochschild cochain complex of $A$ with coefficients in $V$ as

$$
\mathrm{CH}_{\text {Hopf }}^{*}(A, V):=\operatorname{Hom}_{A^{e} \rtimes B}\left(\mathrm{CB}_{*}(A), V\right) .
$$

$H H_{\text {Hopf }}^{*}(A, V)$, the Hopf-Hochschild cohomology of $A$ with coefficients in $V$, is defined to be the cohomology of this cochain complex.

Definition 3.7. Let $A$ be a non-unital associative algebra and let $\Omega(A)$ be the kernel of the multiplication map $\mu: A^{\otimes 2} \rightarrow A$. We define the shifted brutal truncation $\mathrm{CB}_{*}(A)_{>0}$ as the differential graded $k$-module given by $\mathrm{CB}_{n}(A)_{>0}:=\mathrm{CB}_{n+1}(A)$ for $n \geqslant 0$ with the shifted differentials $d_{*+1}^{\mathrm{CB}}$. A non-unital $k$-algebra $A$ is called $H$-unital if the homology of $\mathrm{CB}_{*}(A)_{>0}$ is concentrated at degree 0 and $H_{0}\left(\mathrm{CB}_{*}(A)_{>0}\right) \cong$ $\Omega(A)$.

Remark 3.8. The classical definition of an $H$-unital algebra $A$ by Wodzicki $[\mathbf{2 0}]$ is that the standard Bar resolution of $A$ is acyclic. However, one can easily show that the definition we gave above is equivalent to Wodzicki's definition.

Theorem 3.9. Let $A$ be an $H$-unital projective $B$-module algebra and assume $V$ is an arbitrary $B$-equivariant $A$-bimodule. Then one has isomorphisms of the form

$$
H H_{\mathrm{Hopf}}^{n+1}(A, V) \cong \operatorname{Ext}_{A^{e} \rtimes B}^{n}(\Omega(A), V)
$$

for any $n \geqslant 1$.

Proof. We have a short exact sequence of $A^{e} \rtimes B$-modules of the form

$$
0 \rightarrow \Omega(A) \rightarrow A^{e} \stackrel{\mu_{A}}{\longrightarrow} A \rightarrow 0
$$

where $\mu_{A}$ denotes the multiplication morphism. If $A$ is a free $B$-module, then $A^{\otimes n}$ is also a free $B$-module for any $n \geqslant 1$ provided $B$ is a Hopf algebra. This means if $A$ is $B$-projective, then so are $A^{\otimes n}$ for $n \geqslant 1$. This, in turn, implies $A^{\otimes n}$ is a projective $A^{e} \rtimes B$-module for $n \geqslant 3$. Now, since $A$ is $B$-projective and $H$-unital, the shifted brutal truncation $\mathrm{CB}_{*}(A)_{>0}$ is a $A^{e} \rtimes B$-projective resolution of $\Omega(A)$ the kernel of the multiplication map. The result follows.

Definition 3.10. Assume $A$ is a $B$-module algebra and $V$ is a $B$-equivariant $A$ module. A morphism of $k$-modules $A \stackrel{D}{\longrightarrow} V$ is called a $V$-valued derivation on $A$ if and only if

$$
D\left(a a^{\prime}\right)=D(a) a^{\prime}+a D\left(a^{\prime}\right)
$$

for any $a, a^{\prime} \in A$. The same derivation is called a $V$-valued $B$-equivariant derivation if

$$
D(b a)=b D(a)
$$

for any $a \in A$ and $b \in B$. The $k$-module of $V$-valued and $V$-valued $B$-equivariant derivations on $A$ are denoted by $\operatorname{Der}(A, V)$ and $\operatorname{Der}_{B}(A, V)$ respectively. 
Let $v \in V$ be fixed and consider the $k$-module morphism $A \stackrel{[v, \cdot]}{\longrightarrow} V$ defined by $[v, a]=v \triangleleft a-a \triangleright v$ for any $a \in A$. Then

$$
\left[v, a a^{\prime}\right]=v \triangleleft a a^{\prime}-a a^{\prime} \triangleright v=[v, a] \triangleleft a^{\prime}+a \triangleright\left[v, a^{\prime}\right]
$$

for any $a, a^{\prime} \in A$ and $b \in B$. This means that the elements of the form $[v, \cdot]$ in $\operatorname{Hom}_{k}(A, V)$ are $V$-valued derivations on $A$. However if we were to require $[v, \cdot]$ to be a $B$-equivariant derivation, then we need to have

$$
[v, b(a)]=v \triangleleft(b a)-(b a) \triangleright v=\left(b_{(1)} v\right) \triangleleft\left(b_{(2)} a\right)-\left(b_{(1)} a\right) \triangleright\left(b_{(2)} v\right)=b[v, a]
$$

for any $a \in A, b \in B$. In case $V$ is a trivial $B$-module via the counit, i.e. $b(v)=\varepsilon(b) v$, then the condition above is satisfied.

For a left $A^{e} \rtimes B$-module $V$, we let ${ }^{B} V:=\{v \in V \mid b(v)=\varepsilon(b) v, b \in B\}$ of $V$ and $V^{\operatorname{Lie}(A)}:=\{v \in V \mid a \triangleright v-v \triangleleft a=0$, for any $a \in A\}$.

Proposition 3.11. Let $A$ be an B-module algebra and $V$ be an B-equivariant $A$ module as before. Then one has

$$
H H_{\mathrm{Hopf}}^{0}(A, V) \cong\left({ }^{B} V\right)^{\operatorname{Lie}(A)} \quad \text { and } \quad H H_{\mathrm{Hopf}}^{1}(A, V) \cong \operatorname{Der}_{B}(A, V) /\left[A,{ }^{B} V\right] .
$$

Proof. If we consider $f \in \operatorname{Hom}_{A^{e} \rtimes B}\left(\mathrm{CB}_{n}(A), V\right)$, we see that

$$
f\left(a_{0} \otimes \cdots \otimes a_{n+1}\right)=a_{0} f\left(1 \otimes a_{1} \otimes \cdots \otimes a_{n} \otimes 1\right) a_{n+1} .
$$

Since $b\left(1_{A}\right)=\varepsilon(b) 1_{A}$ for all $b \in B$, we see that $\mathrm{CH}_{\mathrm{Hopf}}^{0}(A, V) \cong \operatorname{Hom}_{B}(k, V) \cong B V$. The proof that $\mathrm{CH}_{*}^{1}(A, V) \cong \operatorname{Hom}_{B}(A, V)$ is similar. Then $v \in H H_{\text {Hopf }}^{0}(A, V)$ if and only if

$$
d_{\mathrm{CH}}^{0}(v)(1 \otimes a \otimes 1)=v(a \otimes 1)-v(1 \otimes a)=a \triangleright v-v \triangleleft a=0,
$$

i.e. $[a, v]=0$ for any $a \in A$, which is the same as the invariants of the adjoint action of $\operatorname{Lie}(A)$ on ${ }^{B} V$; i.e. $\left({ }^{B} V\right)^{\operatorname{Lie}(A)}$. Similarly, $f \in \operatorname{ker}\left(d_{\mathrm{CH}}^{1}\right)$ if and only if

$$
\begin{aligned}
\left(d_{\mathrm{CH}}^{1} f\right)\left(1 \otimes a \otimes a^{\prime} \otimes 1\right) & =f\left(a \otimes a^{\prime} \otimes 1\right)-f\left(1 \otimes a a^{\prime} \otimes 1\right)+f\left(1 \otimes a \otimes a^{\prime}\right) \\
& =a f\left(1 \otimes a^{\prime} \otimes 1\right)-f\left(1 \otimes a a^{\prime} \otimes 1\right)+f(1 \otimes a \otimes 1) a^{\prime}=0
\end{aligned}
$$

for any $\left(1 \otimes a \otimes a^{\prime} \otimes 1\right)$ in $\mathrm{CB}_{2}(A)$. In other words, $f \in \operatorname{ker}\left(d_{\mathrm{CH}}^{1}\right)$ if and only if $D_{f}(a):=f(1 \otimes a \otimes 1)$ is a derivation. Moreover, since $f$ is $B$-equivariant, so is $D_{f}$. Equation 3.1 tells us that the image of $d_{\mathrm{CH}}^{0}$ consists of the elements of the form $[a, v]$ where $v \in{ }^{B} V$ and $a \in A$. In other words, $H H_{\text {Hopf }}^{1}(A, V) \cong \operatorname{Der}_{B}(A, V) /\left[A,{ }^{B} V\right]$.

\section{Hopf-Hochschild homology revisited}

Definition 4.1. Assume $B$ is a Hopf algebra with an invertible antipode and let $U$ be a left $A^{e} \rtimes B$-module. Then one can think of $U$ as a right $A^{e} \rtimes B$-module (denoted by $U^{o p}$ ) via the action

$$
u^{o p}\left(a \otimes a^{\prime} \otimes 1_{B}\right):=\left(a^{\prime} \triangleright u \triangleleft a\right)^{o p} \quad \text { and } \quad u^{o p}\left(1_{A} \otimes 1_{A} \otimes b\right):=\left(S^{-1}(b) u\right)^{o p}
$$


for any $u^{o p} \in U^{o p}, a, a^{\prime} \in A$ and $b \in B$. In order this definition to yield a right $A^{e} \rtimes B$-module structure, one must have

$$
\begin{aligned}
\left(u^{o p}\right. & \left.\left(1_{A} \otimes 1_{A} \otimes b\right)\right)\left(a \otimes a^{\prime} \otimes 1_{B}\right) \\
& =\left(a^{\prime} \triangleright\left(S^{-1}(b) u\right) \triangleleft a\right)^{o p} \\
& =\left(S\left(b_{(2)(3)}\right) b_{(3)}\left(a^{\prime}\right) \triangleright S^{-1}\left(b_{(2)(2)}\right) u \triangleleft S^{-1}\left(b_{(2)(1)}\right) b_{(1)}(a)\right)^{o p} \\
& =u^{o p}\left(b_{(1)}(a) \otimes b_{(3)}\left(a^{\prime}\right) \otimes b_{(2)}\right)
\end{aligned}
$$

which follows from the identity

$$
\left(a \otimes a^{\prime} \otimes b\right)=\left(1 \otimes 1 \otimes b_{(2)}\right)\left(S^{-1}\left(b_{(1)}\right)(a) \otimes S\left(b_{(3)}\right)\left(a^{\prime}\right) \otimes 1_{B}\right)
$$

for any $a, a^{\prime} \in A$ and $b \in B$.

Theorem 4.2. Assume $B$ is a Hopf algebra with an invertible antipode. Let $A$ be a B-module algebra and $V$ be a left $A^{e} \rtimes B$-module. Then ${ }_{B} \mathrm{QCH}_{*}(A, B, V)$ and $V^{\text {op }} \underset{A^{e} \rtimes B}{\otimes} \mathrm{CB}_{*}(A)$ are isomorphic as differential graded $k$-modules.

Proof. Define a morphism of graded modules $\varphi_{*}: \mathrm{CH}_{*}(A, V) \rightarrow V^{o p} \underset{A^{e} \rtimes B}{\otimes} \mathrm{CB}_{*}(A)$ by letting

$$
\varphi_{n}\left(v \otimes a_{1} \otimes \cdots \otimes a_{n}\right):=v^{o p} \underset{A^{e} \rtimes B}{\otimes}\left(1 \otimes a_{1} \otimes \cdots \otimes a_{n} \otimes 1\right)
$$

for any $\left(v \otimes a_{1} \otimes \cdots \otimes a_{n}\right) \in \mathrm{CH}_{n}(A, V)$. One can easily check that $\varphi_{*}$ is a morphism of pre-simplicial $k$-modules, and therefore also a morphism of differential graded $k$ modules. Notice that we have

$$
\begin{aligned}
\varphi_{n} L_{b}\left(v \otimes a_{1} \otimes \cdots \otimes a_{n}\right) & =v^{o p} S\left(b_{(1)}\right) \underset{A^{e} \rtimes B}{\otimes}\left(1 \otimes L_{b_{(2)}}\left(a_{1} \otimes \cdots \otimes a_{n}\right) \otimes 1\right) \\
& =v^{o p} S\left(b_{(1)}\right) \underset{A^{e} \rtimes B}{\otimes} L_{b_{(2)}}\left(1 \otimes a_{1} \otimes \cdots \otimes a_{n} \otimes 1\right) \\
& =\varepsilon(b) v \underset{A^{e} \rtimes B}{\otimes}\left(1 \otimes a_{1} \otimes \cdots \otimes a_{n} \otimes 1\right) \\
& =\varepsilon(b) \varphi_{n}\left(v \otimes a_{1} \otimes \cdots \otimes a_{n}\right)
\end{aligned}
$$

by observing $b\left(1_{A}\right)=\varepsilon(b) 1_{A}$ for any $b \in B$. This means $\varphi_{*}$ is a morphism of graded $B$-modules where we think of the codomain as a graded $B$-module via the trivial action. Moreover, for any $b \in B$ we also have

$$
\varphi_{n}\left[L_{b}, \partial_{n+1}\right]=\varphi_{n} L_{b} \partial_{n+1}-\varphi_{n} \partial_{n+1} L_{b}=\varphi_{n} \partial_{n+1}\left(\varepsilon(b)-L_{b}\right) .
$$

Together with the fact that $\varphi_{*}$ is a morphism of pre-simplicial $k$-modules we see

$$
\varphi_{n}\left[L_{b}, \partial_{n+1}\right]=\left(i d_{V} \underset{A^{e} \rtimes B}{\otimes} \partial_{n+1}\right) \varphi_{n+1}\left(\varepsilon(b)-L_{b}\right)=0
$$

for any $b \in B$. Recall that $\mathrm{QCH}_{*}(A, B, V)$ is obtained from $\mathrm{CH}_{*}(A, V)$ by dividing out the graded $B$-submodule and the pre-simplicial $k$-submodule $J_{*}(A, B, V)$ generated by elements of the form $\left[L_{b}, \partial_{n+1}\right] \Psi$, where $b \in B, \Psi \in \mathrm{CH}_{n}(A, V)$ and $n \geqslant 0$. Since $\varphi_{*}$ annihilates the submodule $J_{*}(A, B, V)$ we divide out, we get an exten$\operatorname{sion} \varphi_{*}^{\prime}: \mathrm{QCH}_{*}(B, A, V) \rightarrow V^{o p} \underset{A^{e} \rtimes B}{\otimes} \mathrm{CB}_{*}(A)$ which is a morphism of pre-simplicial 
and differential graded $B$-modules. However, the codomain is a trivial $B$-module. Therefore we get a commutative diagram of the form

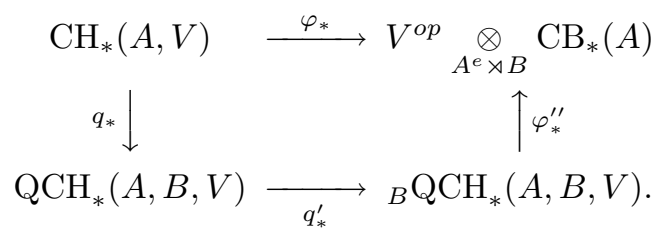

Since $\varphi_{*}$ is a morphism of differential graded $k$-modules so is $\varphi_{*}^{\prime \prime}$ because both $q_{*}$ and $q_{*}^{\prime}$ are epimorphisms of differential graded $k$-modules. Now define a morphism of graded $k$-modules

$$
V^{o p} \underset{A^{e} \rtimes B}{\otimes} \mathrm{CB}_{*}(A) \stackrel{s_{*}}{\longrightarrow}{ }_{B} \mathrm{QCH}_{*}(A, B, V),
$$

by letting

$$
s_{n}\left(v^{o p} \underset{A^{e} \rtimes B}{\otimes}\left(1 \otimes a_{1} \otimes \cdots \otimes a_{n} \otimes 1\right)\right)=\left[v \otimes a_{1} \otimes \cdots \otimes a_{n}\right]
$$

for any $v^{o p} \underset{A^{e} \rtimes B}{\otimes}\left(1 \otimes a_{1} \otimes \cdots \otimes a_{n} \otimes 1\right)$ in $V^{o p} \underset{A^{e} \rtimes B}{\otimes} \mathrm{CB}_{*}(A)$. Here we use the notation $[\Psi]$ to denote the class of an element $\Psi \in \mathrm{CH}_{*}(A, V)$ in ${ }_{B} \mathrm{QCH}_{*}(A, V)$. The morphism $s_{*}$ is well-defined since

$$
\begin{aligned}
s_{n} & \left(v^{o p} \underset{A^{e} \rtimes B}{\otimes}\left(a \otimes a^{\prime} \otimes 1_{B}\right)\left(1 \otimes a_{1} \otimes \cdots \otimes a_{n} \otimes 1\right)\right) \\
& =\left[a^{\prime} \triangleright v \triangleleft a \otimes a_{1} \otimes \cdots \otimes a_{n}\right] \\
& =s_{n}\left(v^{o p}\left(a \otimes a^{\prime} \otimes 1_{B}\right) \underset{A^{e} \rtimes B}{\otimes}\left(1 \otimes a_{1} \otimes \cdots \otimes a_{n} \otimes 1\right)\right),
\end{aligned}
$$

and

$$
\begin{aligned}
s_{n} & \left(v^{o p} \underset{A^{e} \rtimes B}{\otimes}\left(1_{A} \otimes 1_{A} \otimes b\right)\left(1 \otimes a_{1} \otimes \cdots \otimes a_{n} \otimes 1\right)\right) \\
& =\left[v \otimes b_{(1)}\left(a_{1}\right) \otimes \cdots \otimes b_{(n)}\left(a_{n}\right)\right] \\
& =\left[b_{(2)} S^{-1}\left(b_{(1)}\right) v \otimes b_{(3)}\left(a_{1}\right) \otimes \cdots \otimes b_{(n+2)}\left(a_{n}\right)\right] \\
& =\left[S^{-1}(b) v \otimes a_{1} \otimes \cdots \otimes a_{n}\right] \\
& =s_{n}\left(v^{o p}\left(1_{A} \otimes 1_{A} \otimes b\right) \underset{A^{e} \rtimes B}{\otimes}\left(1 \otimes a_{1} \otimes \cdots \otimes a_{n} \otimes 1\right)\right)
\end{aligned}
$$

for any $a, a^{\prime} \in A, b \in B$ and $v^{o p} \underset{A^{e} \rtimes B}{\otimes}\left(1 \otimes a_{1} \otimes \cdots \otimes a_{n} \otimes 1\right)$ in $V^{o p} \underset{A^{e} \rtimes B}{\otimes} \mathrm{CB}_{*}(A)$. One can easily check that $s_{*} \varphi_{*}^{\prime \prime}=i d_{*}=\varphi_{*}^{\prime \prime} s_{*}$. The result follows.

Corollary 4.3. Let $B$ be a Hopf algebra with an invertible antipode. Assume $A$ is a B-module algebra and $V$ is a left $B$-equivariant $A$-bimodule. Then

$$
H H_{0}^{\mathrm{Hopf}}\left(A, V^{o p}\right) \cong{ }_{B} V /\left[A,{ }_{B} V\right]
$$

where ${ }_{B} V:=k \underset{B}{\otimes} V \cong A^{e} \underset{A^{e} \rtimes B}{\otimes} V$. 
Corollary 4.4. Let $B$ be a Hopf algebra with an invertible antipode. Assume $A$ is an $H$-unital projective $B$-module algebra and $V$ is a $B$-equivariant $A$-bimodule. Then one has isomorphisms of the form

$$
H H_{n+1}^{\mathrm{Hopf}}(A, V) \cong \operatorname{Tor}_{n}^{A^{e} \rtimes B}\left(\Omega(A), V^{o p}\right)
$$

for any $n \geqslant 1$.

Proof. Consider the short exact sequence of $A^{e} \rtimes B$-modules

$$
0 \rightarrow \Omega(A) \rightarrow A^{e} \stackrel{\mu_{A}}{\longrightarrow} A \rightarrow 0,
$$

where $\mu_{A}$ is the multiplication on $A$. If $A$ is $H$-unital and $A$ is $B$-projective then the shifted brutal truncation $\mathrm{CB}_{*}(A)_{>0}$ of $\mathrm{CB}_{*}(A)$ is a $A^{e} \rtimes B$-projective resolution of $\Omega(A)$. The rest of the proof is similar to that of Theorem 3.9.

Remark 4.5. After Theorem 4.2 and Corollary 4.4, one can see that there is another possible definition of the Hopf-Hochschild complex of a $B$-module algebra $A$. Namely, one can use the differential graded $k$-module $V \underset{A^{e} \rtimes B}{\otimes} \mathrm{CB}_{*}(A)$ to define the Hopf-Hochschild homology of $A$ for a right $B$-equivariant $A$-bimodule $V$.

\section{Categorical algebra and cofinality}

Definition 5.1. A small category $\mathcal{C}$ is called $k$-linear if for each $X, Y \in O b(\mathcal{C})$, the Hom object $\operatorname{Hom}_{\mathcal{C}}(X, Y)$ is a $k$-module and the composition maps

$$
\operatorname{Hom}_{\mathcal{C}}(Y, Z) \times \operatorname{Hom}_{\mathcal{C}}(X, Y) \rightarrow \operatorname{Hom}_{\mathcal{C}}(X, Z)
$$

are $k$-bilinear for any $X, Y, Z \in O b(\mathcal{C})$. A functor $\mathcal{C} \stackrel{F}{\longrightarrow} \mathcal{C}^{\prime}$ between two $k$-linear categories is called a $k$-linear functor if the map

$$
\operatorname{Hom}_{\mathcal{C}}(X, Y) \stackrel{F_{X, Y}}{\longrightarrow} \operatorname{Hom}_{\mathcal{C}^{\prime}}(X, Y)
$$

is a morphism of $k$-modules for any $X, Y$ in $O b(\mathcal{C})$.

Definition 5.2. A $k$-linear bifunctor on $\mathcal{C}$ with values in another $k$-linear category $\mathcal{C}^{\prime}$ is just a $k$-linear functor of the form $\mathcal{C} \times \mathcal{C}^{o p} \stackrel{\mathcal{H}}{\longrightarrow} \mathcal{C}^{\prime}$.

Definition 5.3. Let $\mathcal{C}$ be a $k$-linear small category and let $\mathcal{H}$ be a bifunctor on $\mathcal{C}$ with values in $k$-Mod. Define $\mathrm{CH}_{*}(\mathcal{C}, \mathcal{H})$, the Hochschild complex of $\mathcal{C}$ with coefficients in the bifunctor $\mathcal{H}$, as the differential graded $k$-module given by

$$
\mathrm{CH}_{n}(\mathcal{C}, \mathcal{H}):=\bigoplus_{X_{0}, \ldots, X_{n}} \mathcal{H}\left(X_{0}, X_{n}\right) \otimes \operatorname{Hom}_{\mathcal{C}}\left(X_{1}, X_{0}\right) \otimes \cdots \otimes \operatorname{Hom}_{\mathcal{C}}\left(X_{n}, X_{n-1}\right)
$$

with a pre-simplicial structure

$$
\begin{aligned}
& \partial_{j}\left(h \otimes X_{0} \stackrel{u_{1}}{\longleftarrow} \cdots \stackrel{u_{n}}{\longleftarrow} X_{n}\right) \\
& = \begin{cases}\mathcal{H}\left(u_{1}, i d_{X_{n}}\right)(h) \otimes X_{1} \stackrel{u_{2}}{\longleftarrow} \cdots \stackrel{u_{n}}{\longleftarrow} X_{n}, & \text { if } j=0 \\
h \otimes \cdots \stackrel{u_{i-1}}{\longleftarrow} X_{i-1} \stackrel{u_{i} u_{i+1}}{\longleftarrow} X_{i+1} \stackrel{u_{i+2}}{\longleftarrow} \cdots, & \text { if } 0<j<n-1 \\
\mathcal{H}\left(i d_{X_{0}}, u_{n}\right)(h) \otimes X_{0} \stackrel{u_{1}}{\longleftarrow} \cdots X_{n-1}, & \text { if } j=n\end{cases}
\end{aligned}
$$


defined for any $n \geqslant 1$ and $\left(h \otimes X_{0} \stackrel{u_{1}}{\longleftarrow} \ldots \stackrel{u_{n}}{\longleftarrow} X_{n}\right)$ in $\mathrm{CH}_{n}(\mathcal{C}, \mathcal{H})$. In the case $\mathcal{H}=$ $\operatorname{Hom}_{\mathcal{C}}$, we denote $\mathrm{CH}_{*}\left(\mathcal{C}, \operatorname{Hom}_{\mathcal{C}}\right)$ simply by $\mathrm{CH}_{*}(\mathcal{C})$.

Assume $\mathcal{A}_{*}$ and $\mathcal{B}_{*}$ are two pre-simplicial $k$-modules and let $f, g: \mathcal{A}_{*} \rightarrow \mathcal{B}_{*}$ be two morphisms of pre-simplicial modules. Now, recall from $[\mathbf{1 6}]$ that a pre-simplicial homotopy $h_{*}$ between $f_{*}$ and $g_{*}$ is a set of $k$-module morphisms $h_{i}: \mathcal{A}_{n} \rightarrow \mathcal{B}_{n+1}$ defined for $0 \leqslant i \leqslant n$ satisfying

$$
h_{i} \partial_{j}=\partial_{j} h_{i+1}, \text { if } j \leqslant i, \quad h_{i} \partial_{j}=\partial_{j+1} h_{i}, \text { if } j \geqslant i+1, \quad \partial_{i} h_{i}=\partial_{i} h_{i-1},
$$

where $f_{*}=\partial_{0} h_{0}$ and $g_{*}=\partial_{*+1} h_{*}$.

Definition 5.4. Let $\mathcal{C}$ be a $k$-linear category and let $\mathcal{D}$ be a $k$-linear subcategory of $\mathcal{C}$. Then $\mathcal{D}$ is called a cofinal subcategory of $\mathcal{C}$ if for every object $C$ of $\mathcal{C}$, there exists an object $D$ in $\mathcal{D}$ and a retract $D \stackrel{r}{\rightarrow} C$ in $\mathcal{C}$.

Theorem 5.5. Let $\mathcal{C}$ be a small $k$-linear category and $\mathcal{H}$ be a bifunctor on $\mathcal{C}$ with values in $k$-Mod. Assume $\mathcal{D}$ is a cofinal subcategory of $\mathcal{C}$. Then the natural inclusion $\mathrm{CH}_{*}(\mathcal{D}, \mathcal{H}) \stackrel{i_{*}}{\longrightarrow} \mathrm{CH}_{*}(\mathcal{C}, \mathcal{H})$ is a homotopy equivalence.

Proof. For every object $C$ in $\mathcal{C}$ fix a choice of object $\delta(C)$ and a retract $\delta(C) \stackrel{r(C)}{\longrightarrow} C$ such that for each object $D$ in $\mathcal{D}$, the choice is $D \stackrel{i d_{D}}{\longrightarrow} D$. Denote the left inverse of $r(C)$ by $s(C)$ for every $C$ in $\mathcal{C}$. Now define a morphism of differential graded $k$-modules $\mathrm{CH}_{*}(\mathcal{C}, \mathcal{H}) \stackrel{M_{*}}{\longrightarrow} \mathrm{CH}_{*}(\mathcal{D}, \mathcal{H})$ by letting

$$
M_{*}\left(h \otimes X_{0} \stackrel{u_{1}}{\longleftarrow} \cdots \stackrel{u_{n}}{\longleftarrow} X_{n}\right)=\mathcal{H}\left(s_{n}, r_{0}\right)(h) \otimes \delta\left(X_{0}\right) \stackrel{s_{0} u_{1} r_{1}}{\longleftarrow} \cdots \stackrel{s_{n-1} u_{n} r_{n}}{\longleftarrow} \delta\left(X_{n}\right)
$$

for any $\left(h \otimes X_{0} \stackrel{u_{1}}{\longleftarrow} \ldots \stackrel{u_{n}}{\longleftarrow} X_{n}\right)$ in $\mathrm{CH}_{n}(\mathcal{C}, \mathcal{H})$ where we use $r_{i}=r\left(X_{i}\right)$ and $s_{i}=$ $s\left(X_{i}\right)$ for any $0 \leqslant i \leqslant n$. It is easy to see that $M_{*}$ is a morphism of pre-simplicial modules since $r_{i} s_{i}=i d_{i}$. Note that the composition $M_{*} i_{*}$ is identity on $\mathrm{CH}_{*}(\mathcal{D}, \mathcal{H})$. We are going to show $i_{*} M_{*}$ is homotopic to the identity on $\mathrm{CH}_{*}(\mathcal{C}, \mathcal{H})$. We define a pre-simplicial homotopy by letting

$$
\begin{aligned}
& h_{i}\left(h \otimes X_{0} \stackrel{u_{1}}{\longleftarrow} \cdots \stackrel{u_{n}}{\longleftarrow} X_{n}\right) \\
& =\mathcal{H}\left(s_{n}, i d_{X_{0}}\right)(h) \otimes X_{0} \stackrel{u_{1}}{\longleftarrow} \cdots \stackrel{u_{i}}{\longleftarrow} X_{i} \stackrel{r_{i}}{\longleftarrow} \delta\left(X_{i}\right) \stackrel{s_{i} u_{i+1} r_{i+1}}{\longleftarrow} \cdots \stackrel{s_{n-1} u_{n} r_{n}}{\longleftarrow} \delta\left(X_{n}\right)
\end{aligned}
$$

for any $0 \leqslant i \leqslant n$ and for any $\left(h \otimes X_{0} \stackrel{u_{1}}{\longleftarrow} \cdots \stackrel{u_{n}}{\longleftarrow} X_{n}\right)$ in $\mathrm{CH}_{*}(\mathcal{C}, \mathcal{H})$. Note that $\partial_{0} h_{0}=i_{*} M_{*}$ and $\partial_{n+1} h_{n}=i d_{*}$. We leave the verification of pre-simplicial homotopy identities to the reader.

Corollary 5.6. If $\mathcal{C}^{\prime} \stackrel{F}{\longrightarrow} \mathcal{C}$ is a k-linear functor, then one has a morphism of differential graded $k$-modules of the form

$$
\mathrm{CH}_{*}\left(\mathcal{C}^{\prime}, \mathcal{H F}\right) \stackrel{F_{*}}{\longrightarrow} \mathrm{CH}_{*}(\mathcal{C}, \mathcal{H})
$$

for any bifunctor $\mathcal{H}$ on $\mathcal{C}$ with values in $k$-Mod. Moreover, if $F$ is an equivalence of categories then $F_{*}$ is a homotopy equivalence.

Proof. First, let us explain what $\mathrm{CH}_{*}\left(\mathcal{C}^{\prime}, \mathcal{H F}\right)$ is: $\mathrm{CH}_{n}\left(\mathcal{C}^{\prime}, \mathcal{H F}\right)$ generated by homogeneous tensors of the form $\left(h \otimes X_{0} \stackrel{u_{1}}{\longleftarrow} \cdots \stackrel{u_{n}}{\longleftarrow} X_{n}\right)$, where $h \in \mathcal{H}\left(F\left(X_{n}\right), F\left(X_{0}\right)\right)$ 
for any $n \geqslant 0$. The "action" of $u_{1}$ and $u_{n}$ on $h$ is defined through $F$. The morphism $F_{*}$ of pre-simplicial $k$-modules is defined as

$$
F_{n}\left(h \otimes X_{0} \stackrel{u_{1}}{\longleftarrow} \cdots \stackrel{u_{n}}{\longleftarrow} X_{n}\right)=h \otimes F\left(X_{0}\right) \stackrel{F\left(u_{1}\right)}{\longleftarrow} \cdots \stackrel{F\left(u_{n}\right)}{\longleftarrow} F\left(X_{n}\right)
$$

for any $n \geqslant 0$ and $\left(h \otimes X_{0} \stackrel{u_{1}}{\longleftarrow} \ldots \stackrel{u_{n}}{\longleftarrow} X_{n}\right)$ in $\mathrm{CH}_{n}\left(\mathcal{C}^{\prime}, \mathcal{H} F\right)$. Now assume $F$ is an equivalence with a quasi-inverse $G$ and with the isomorphism $i d_{\mathcal{C}^{\prime}} \stackrel{\varphi}{\longrightarrow} G F$. Note that we have the composition

$$
\mathrm{CH}_{*}\left(\mathcal{C}^{\prime}, \mathcal{H} F G F\right) \stackrel{G_{*} F_{*}}{\longrightarrow} \mathrm{CH}_{*}\left(\mathcal{C}^{\prime}, \mathcal{H} F\right)
$$

and the image of the functor $\mathcal{C}^{\prime} \stackrel{G F}{\longrightarrow} \mathcal{C}^{\prime}$ is a cofinal subcategory of $\mathcal{C}^{\prime}$ since $G F \simeq i d_{\mathcal{C}^{\prime}}$. Thus $G_{*} F_{*} \simeq i d_{*}$. The same argument works also for $\mathcal{C} \stackrel{F G}{\longrightarrow} \mathcal{C}$ and we see that $F_{*} G_{*} \simeq i d_{*}$. The result follows.

Definition 5.7. Let $\mathcal{C}$ be a $k$-linear category which has finite coproducts and let $\mathcal{D}$ and $\mathcal{E}$ be two full $k$-linear subcategories. $\mathcal{D}$ is said to generate $\mathcal{E}$ if for every object $E$ of $\mathcal{E}$ there is a natural number $n \geqslant 1$ and a set of objects $D_{1}, \ldots, D_{n}$ of $D$ such that $E \cong \coprod_{i=1}^{n} D_{i}$.

Theorem 5.8. Let $\mathcal{C}, \mathcal{D}$ and $\mathcal{E}$ be as in Definition 5.7. Then the natural inclusion $\mathrm{CH}_{*}(\mathcal{D}, \mathcal{H}) \stackrel{i_{*}}{\longrightarrow} \mathrm{CH}_{*}(\mathcal{E}, \mathcal{H})$ is a homotopy equivalence for any bifunctor $\mathcal{H}$ on $\mathcal{C}$ with values in $k$-Mod.

Proof. Take an object $E$ from $\mathcal{E}$ and consider "D-components" $\left\{D_{1}, \ldots, D_{n}\right\}$ of $E$. Since $E \cong \coprod_{i=1}^{n} D_{i}$ and

$$
\operatorname{Hom}_{\mathcal{C}}(E, E) \cong \bigoplus_{i, j} \operatorname{Hom}_{\mathcal{C}}\left(D_{i}, D_{j}\right)
$$

there are morphisms $E \stackrel{v_{i}}{\longleftarrow} D_{i}$ and $D_{j} \stackrel{u_{j}}{\longleftarrow} E$ such that $\sum_{i} v_{i} u_{i}=i d_{E}$. Now take $h \otimes$ $E_{0} \stackrel{f_{1}}{\longleftarrow} \ldots \stackrel{f_{n}}{\longleftarrow} E_{n}$ in $\mathrm{CH}_{n}(\mathcal{E}, \mathcal{H})$ and let $D_{j}^{i}$ be the $\mathcal{D}$-components of $E_{i}$, and $E_{i} \stackrel{v_{j}^{i}}{\longleftarrow}$ $D_{j}^{i} \stackrel{u_{j}^{i}}{\longleftarrow} E_{i}$ be the corresponding splitting of $i d_{E_{i}}$ for $0 \leqslant i \leqslant n$. Define a morphism of pre-simplicial modules $\mathrm{CH}_{*}(\mathcal{E}, \mathcal{H}) \stackrel{M_{*}}{\longrightarrow} \mathrm{CH}_{*}(\mathcal{D}, \mathcal{H})$ by letting

$$
\begin{aligned}
M_{n}\left(h \otimes E_{0} \stackrel{f_{1}}{\longleftarrow} \cdots \stackrel{f_{n}}{\longleftarrow} E_{n}\right) \\
\quad=\sum_{i_{0}, \ldots, i_{n}} \mathcal{H}\left(u_{i_{n}}^{n}, v_{i_{0}}^{0}\right)(h) \otimes D_{i_{0}}^{0} \stackrel{u_{i_{0}}^{0} f_{1} v_{i_{1}}^{1}}{\longleftarrow} \cdots \stackrel{u_{i_{n-1}}^{n-1} f_{n} v_{i_{n}}^{n}}{\longleftarrow} D_{i_{n}}^{n} .
\end{aligned}
$$

Notice that $M_{*} i_{*}$ is the identity on $\mathrm{CH}_{*}(\mathcal{D}, \mathcal{H})$. Observe also that the identity $\sum_{j} v_{j}^{i} u_{j}^{i}=i d_{E_{i}}$ implies $M_{*}$ is a morphism of pre-simplicial modules. Although $i_{*} M_{*}$ is not identity, we will furnish a pre-simplicial homotopy between $i d_{*}$ and $i_{*} M_{*}$ on $\mathrm{CH}_{*}(\mathcal{E}, \mathcal{H})$. We let

$$
\begin{aligned}
& h_{s}\left(h \otimes E_{0} \stackrel{f_{1}}{\longleftarrow} \cdots \stackrel{f_{n}}{\longleftarrow} E_{n}\right) \\
& \quad=\mathcal{H}\left(i d_{E_{n}}, v_{i_{0}}^{0}\right)(h) \otimes D_{i_{0}}^{0} \stackrel{u_{i_{0}}^{0} f_{1} v_{i_{1}}^{1}}{\longleftarrow} \cdots \stackrel{u_{i_{s-1}^{s-1}}^{s} f_{s} v_{i_{s}}^{s}}{\longleftarrow} D_{i_{s}}^{s} \stackrel{u_{i_{s}}^{s}}{\longleftarrow} E_{s} \stackrel{f_{s+1}}{\longleftarrow} \cdots \stackrel{f_{n}}{\longleftarrow} E_{n}
\end{aligned}
$$


for $n \geqslant 0,0 \leqslant s \leqslant n$ and $h \otimes E_{0} \stackrel{f_{1}}{\longleftarrow} \ldots \stackrel{f_{n}}{\longleftarrow} E_{n}$ in $\mathrm{CH}_{n}(\mathcal{E}, \mathcal{H})$. We leave the verification of the pre-simplicial homotopy identities to the reader.

\section{6. $B$-categories and equivariant bifunctors}

Definition 6.1. A $k$-linear category $\mathcal{C}$ is called a $B$-category if each $\operatorname{Hom}_{\mathcal{C}}(X, Y)$ is a left $B$-module and the composition

$$
\operatorname{Hom}_{\mathcal{C}}(Y, Z) \times \operatorname{Hom}_{\mathcal{C}}(X, Y) \rightarrow \operatorname{Hom}_{\mathcal{C}}(X, Z)
$$

is a $B$-module morphism via the diagonal action of $B$ for any $X, Y, Z$ taken from $O b(\mathcal{C})$. In other words, one has $b(g f)=b_{(1)}(g) b_{(2)}(f)$ for any $b \in B, f \in \operatorname{Hom}_{\mathcal{C}}(X, Y)$ and $g \in \operatorname{Hom}_{\mathcal{C}}(Y, Z)$. A functor $\mathcal{C} \stackrel{F}{\longrightarrow} \mathcal{C}^{\prime}$ between two $B$-categories is called $B$-equivariant if the structure morphisms

$$
\operatorname{Hom}_{\mathcal{C}}(X, Y) \stackrel{F_{X, Y}}{\longrightarrow} \operatorname{Hom}_{\mathcal{C}^{\prime}}(F(X), F(Y))
$$

are $B$-module morphisms for any $X, Y \in O b(\mathcal{C})$.

Remark 6.2. It came to our attention that Cibils and Solotar defined the same notion in [6] but they called the same object a $B$-module category. Their primary example of the underlying bialgebra is a group ring which is cocommutative. The bialgebras, or in general Hopf algebras, we consider are not necessarily cocommutative.

Example 6.3. Assume that $B$ is a Hopf algebra and $A$ is a $B$-module algebra. Consider the category $\bmod _{B}-A$ of left $B$-equivariant right $A$-modules and all $A$ linear morphisms. Note that we do consider all $A$-module morphisms, not just $B$ equivariant $A$-module morphisms. Define a left $B$ action of $\operatorname{Hom}_{\bmod _{B}-A}(X, Y)$ by letting

$$
(b f)(x)=b_{(1)} f\left(S\left(b_{(2)}\right) x\right)
$$

for any $f \in \operatorname{Hom}_{\bmod _{B}-A}(X, Y)$ and $x \in X$. However, one needs to show that $b f$ is still a right $A$-module morphism for any $f \in \operatorname{Hom}_{\bmod _{B}-A}(X, Y)$ and $b \in B$. Therefore we check

$$
\begin{aligned}
(b f)(x a) & =b_{(1)} f\left(S\left(b_{(2)}\right)(x a)\right)=b_{(1)} f\left(S\left(b_{(3)}\right)(x) S\left(b_{(2)}\right)(a)\right) \\
& =b_{(1)} f\left(S\left(b_{(4)}\right)(x)\right) b_{(2)} S\left(b_{(3)}\right)(a)=(b f)(x) a
\end{aligned}
$$

for any $a \in A, b \in B, x \in X$ and $f \in \operatorname{Hom}_{\bmod _{B}-A}(X, Y)$. Now notice that for any $g \in \operatorname{Hom}_{\text {mod }_{B}-A}(Y, Z)$ one has

$$
b(g f)(x)=b_{(1)} g f\left(S\left(b_{(2)}\right) x\right)=b_{(1)} g\left(S\left(b_{(2)}\right) b_{(3)} f\left(S\left(b_{(4)}\right) x\right)\right)=\left(b_{(1)} g\right)\left(b_{(2)} f\right)(x)
$$

for any $x \in X$. In other words, $\bmod _{B}-A$ is a $B$-category.

Example 6.4. Assume $B$ is a Hopf algebra and $A$ is a $B$-module algebra. Consider the category $*_{B}^{A}$ which consists of one single object $A$ considered as a right $A$-module via the right regular representation. Then $*_{B}^{A}$ is a $B$-category.

Example 6.5. Assume $B$ is a Hopf algebra and $A$ is a $B$-module algebra. Let free ${ }_{B}-A$ be the subcategory of $\bmod _{B}-A$ generated by arbitrary finite direct sums of $*_{B}^{A}$ by 
itself. Then we define $\operatorname{proj}_{B}-A$ as the full subcategory of $\bmod _{B}-A$ consisting of $A^{e} \rtimes B$-module direct summands of objects in free $B_{B}-A$. Then both free $B_{B} A$ and $\operatorname{proj}_{B}-A$ are $B$-categories.

Definition 6.6. A bifunctor $\mathcal{H}$ on a $B$-category $\mathcal{C}$ with values in $B$-Mod is called a $B$-equivariant bifunctor if the structure morphisms

$$
\mathcal{H}(X, Y) \otimes \operatorname{Hom}_{\mathcal{C}}(Y, Z) \rightarrow \mathcal{H}(X, Z), \quad \operatorname{Hom}_{\mathcal{C}}(W, X) \otimes \mathcal{H}(X, Y) \rightarrow \mathcal{H}(W, Y)
$$

are $B$-module morphisms where $B$ acts diagonally on the left. In other words,

$$
b(\mathcal{H}(u, v)(h))=\mathcal{H}\left(b_{(1)}(u), b_{(3)}(v)\right)\left(b_{(2)}(h)\right)
$$

for any $u \in \operatorname{Hom}_{\mathcal{C}}(W, X), v \in \operatorname{Hom}_{\mathcal{C}}(Y, Z)$ and $h \in \mathcal{H}(X, Y)$, and $b \in B$.

Example 6.7. For a $B$-category $\mathcal{C}$, the bifunctor $\mathcal{H}=\operatorname{Hom}_{\mathcal{C}}(\cdot, \cdot)$ is a $B$-equivariant bifunctor on $\mathcal{C}$ with values in $B$-Mod.

Definition 6.8. For a $B$-category $\mathcal{C}$, let ${ }^{B} \mathcal{C}$ denote the subcategory of morphisms of $\mathcal{C}$ which are $B$-invariant; i.e., $X \stackrel{f}{\rightarrow} Y$ belongs to ${ }^{B} \mathcal{C}$ if and only if $b(f)=\varepsilon(b) f$ for any $b \in B$.

Remark 6.9. Assume $B$ is a Hopf algebra and $\bmod _{B}-A$ be the $B$-category defined in Example 6.3. Then one can see that ${ }^{B} \mathbf{m o d}_{B}$ - $A$, the subcategory of $B$-invariant morphisms, is the category of left $B$-equivariant right $A$-modules and their $B$-equivariant $A$-module morphisms since $b f=\varepsilon(b) f$ if and only if $f$ is $B$-equivariant. We would like to note that for the $B$-equivariant homotopical invariants of $\bmod _{B}-A$ the subcategory ${ }^{B} \mathbf{m o d}_{B}-A$ is rather small. The situation is very similar to topological spaces admitting an action of a fixed group $G$. The $G$-equivariant homotopical invariants of a $G$-space $X$ are computed via $\mathcal{B}(G, X):=E G \bigwedge_{G} X$ rather than $X / G \simeq * \bigwedge_{G} X$. Similarly, ${ }^{B} \bmod _{B}-A$ should be considered as the lowest order equivariant invariant of $\bmod _{B}-A$. Thus we propose that for higher order equivariant homotopical invariants of a $B$-module algebra $A$, such as equivariant $K$-theoretical, Hochschild and cyclic homological invariants, one should use the $B$-category $\bmod _{B}$ - $A$ of $B$-equivariant modules and their $A$-linear morphisms, or its various $B$-subcategories, instead of using simply ${ }^{B} \bmod _{B}$ - $A$ the subcategory of $B$-equivariant $A$-module morphisms of $\bmod _{B}-A$. Our justification lies in Section 7 where we prove Morita invariance in Corollary 7.9.

\section{Morita invariance}

Lemma 7.1. If $\mathcal{C}$ is a $B$-category and $\mathcal{H}$ is a bifunctor on $\mathcal{C}$ with values in $B$-Mod, then $\mathrm{CH}_{*}(\mathcal{C}, \mathcal{H})$ is a graded B-module. However, $\mathrm{CH}_{*}(\mathcal{C}, \mathcal{H})$ is not a pre-simplicial $B$-module.

Proof. The $B$-action of $\mathrm{CH}_{*}(\mathcal{C}, \mathcal{H})$ is defined diagonally; i.e.,

$$
L_{b}\left(h \otimes X_{0} \stackrel{u_{1}}{\longleftarrow} \cdots \stackrel{u_{n}}{\longleftarrow} X_{n}\right):=b_{(1)}(h) \otimes X_{0} \stackrel{b_{(2)} u_{1}}{\longleftarrow} \cdots \stackrel{b_{(n+1)} u_{n}}{\longleftarrow} X_{n}
$$

for any $b \in B, n \geqslant 0$ and $h \otimes X_{0} \stackrel{u_{1}}{\longleftarrow} \cdots \stackrel{u_{n}}{\longleftarrow} X_{n}$ in $\mathrm{CH}_{n}(\mathcal{C}, \mathcal{H})$. The fact that presimplicial $k$-module $\mathrm{CH}_{*}(\mathcal{C}, \mathcal{H})$ is NOT a pre-simplicial $B$-module is because of the 
last face morphism: one has

$$
\begin{aligned}
\partial_{n} L_{b}\left(h \otimes X_{0} \stackrel{u_{1}}{\longleftarrow}\right. & \left.\ldots \stackrel{u_{n}}{\longleftarrow} X_{n}\right) \\
& =\mathcal{H}\left(b_{(n+1)}\left(f_{n}\right), i d_{X_{0}}\right)\left(b_{(1)} h\right) \otimes X_{0} \stackrel{b_{(2)} u_{1}}{\longleftarrow} \cdots \stackrel{b_{(n)} u_{n-1}}{\longleftarrow} X_{n-1},
\end{aligned}
$$

which need not be the same as

$$
\begin{aligned}
L_{b} \partial_{n}\left(h \otimes X_{0} \stackrel{u_{1}}{\longleftarrow} \cdots\right. & \left.\stackrel{u_{n}}{\longleftarrow} X_{n}\right) \\
& =\mathcal{H}\left(b_{(1)}\left(f_{n}\right), i d_{X_{0}}\right)\left(b_{(2)} h\right) \otimes X_{0} \stackrel{\stackrel{b_{(3)} u_{1}}{\longleftarrow} \cdots \stackrel{b_{(n+1)} u_{n-1}}{\longleftarrow} X_{n-1}}{ }
\end{aligned}
$$

for any $n \geqslant 0, b \in B$ and $h \otimes X_{0} \stackrel{u_{1}}{\longleftarrow} \ldots \stackrel{u_{n}}{\longleftarrow} X_{n}$ in $\mathrm{CH}_{*}(\mathcal{C}, \mathcal{H})$.

Definition 7.2. Define a graded $k$-submodule $J_{*}(\mathcal{C}, B, \mathcal{H})$ of $\mathrm{CH}_{*}(\mathcal{C}, \mathcal{H})$ generated by elements of the form

$$
\left[L_{b}, \partial_{n}\right]\left(h \otimes X_{0} \stackrel{u_{1}}{\longleftarrow} \cdots \stackrel{u_{n}}{\longleftarrow} X_{n}\right)
$$

where $n \geqslant 1, b \in B$ and $h \otimes X_{0} \stackrel{u_{1}}{\longleftarrow} \cdots \stackrel{u_{n}}{\longleftarrow} X_{n}$ in $\mathrm{CH}_{n}(\mathcal{C}, \mathcal{H})$.

Lemma 7.3. $J_{*}(\mathcal{C}, B, \mathcal{H})$ is a differential graded $k$-submodule and a graded $B$-submodule of $\mathrm{CH}_{*}(\mathcal{C}, \mathcal{H})$. Therefore, $\mathrm{CH}_{*}(\mathcal{C}, \mathcal{H}) / J_{*}(\mathcal{C}, B, \mathcal{H})$ is a differential graded $B$ module.

Proof. The proof is identical to that of Proposition 2.12.

Definition 7.4. Let $\mathrm{QCH}_{*}(\mathcal{C}, B, \mathcal{H})$ be the quotient $\mathrm{CH}_{*}(\mathcal{C}, \mathcal{H}) / J_{*}(\mathcal{C}, B, \mathcal{H})$.

Theorem 7.5. Let $\mathcal{D}$ be a cofinal B-subcategory of $\mathcal{C}$ such that for each $C \in O b(\mathcal{C})$ there is a retract $\delta(C) \stackrel{r(C)}{\longrightarrow} C$ with $\delta(C) \in O b(\mathcal{D})$ and $r(C)$ is B-invariant; i.e. $b(r(C))=\varepsilon(b) r(C)$ for any $b \in B$. Then for any $B$-equivariant bifunctor $\mathcal{H}$ on $\mathcal{C}$ with values in $B$-Mod the natural inclusion

$$
\mathrm{QCH}_{*}(\mathcal{D}, B, \mathcal{H}) \stackrel{i_{*}}{\longrightarrow} \mathrm{QCH}_{*}(\mathcal{C}, B, \mathcal{H})
$$

is a homotopy equivalence.

Proof. The proof is almost verbatim to Theorem 5.5 after noticing $J_{*}(\mathcal{C}, B, \mathcal{H})$ is stable under the pre-simplicial homotopy we furnished there.

Corollary 7.6. Let $\mathcal{C}$ and $\mathcal{C}^{\prime}$ be two $B$-categories and let $\mathcal{C} \stackrel{F}{\rightarrow} \mathcal{C}^{\prime}$ be a functor of $B$-categories. Then for any $B$-equivariant bifunctor $\mathcal{H}$ on $\mathcal{C}^{\prime}$ one has a morphism of differential graded $B$-modules of the form

$$
\mathrm{QCH}_{*}(\mathcal{C}, B, \mathcal{H} F) \stackrel{F_{*}}{\longrightarrow} \mathrm{QCH}_{*}\left(\mathcal{C}^{\prime}, B, \mathcal{H}\right)
$$

Moreover, if $F$ is an equivalence of B-categories, then $F_{*}$ is a homotopy equivalence.

Corollary 7.7. Assume $A$ is a $B$-module algebra. Then $\mathbf{f r e e}_{B}-A$ is a cofinal $B$ subcategory of $\mathbf{p r o j}_{B}-A$. Furthermore, the natural inclusion functor free $\mathbf{f}_{B}-A \rightarrow$ $\operatorname{proj}_{B}-A$ induces a homotopy equivalence of differential graded B-modules of the form

$$
\mathrm{QCH}_{*}\left(\text { free }_{B}-A, B, \mathcal{H}\right) \stackrel{i_{*}}{\longrightarrow} \mathrm{QCH}_{*}\left(\operatorname{proj}_{B}-A, B, \mathcal{H}\right)
$$

for any $B$-equivariant bifunctor $\mathcal{H}$ on $\mathbf{p r o j}_{B}-A$ with values in B-Mod. 
Corollary 7.8. Assume $A$ is a B-module algebra. The subcategory $*_{B}^{A}$ freely generates the $B$-subcategory free $_{B}-A$ of $\operatorname{proj}_{B}-A$. Then the natural inclusion

$$
\mathrm{QCH}_{*}\left(*_{B}^{A}, B, \mathcal{H}\right) \stackrel{i_{*}}{\longrightarrow} \mathrm{QCH}_{*}\left(\text { free }_{B}-A, B, \mathcal{H}\right)
$$

is a homotopy equivalence of differential graded $B$-modules for any $B$-equivariant bifunctor $\mathcal{H}$ defined on proj $_{B}-A$ with values in $B$-Mod. Furthermore, the differential graded $B$-modules $\mathrm{QCH}_{*}\left(*_{B}^{A}, B, \mathcal{H}\right)$ and $\mathrm{QCH}_{*}(A, B, \mathcal{H}(A, A))$ are isomorphic.

Proof. The proof relies on the fact that $\operatorname{Hom}_{\mathbf{p r o j}_{B}-A}(A, A) \cong A$. The rest of the proof is trivial.

Theorem 7.9 (Morita invariance for Hopf-Hochschild (co)homology). Let $B$ be a Hopf algebra and assume that $A$ and $A^{\prime}$ are two $B$-module algebras. If $\bmod _{B}-A$ and $\bmod _{B}-A^{\prime}$ the category of finitely generated $B$-equivariant representations of $A$ and $A^{\prime}$ respectively are $B$-equivariantly equivalent, then Hopf-Hochschild complex ${ }_{B} \mathrm{QCH}_{*}(A, B, A)$ of $A$ and Hopf-Hochschild complex ${ }_{B} \mathrm{QCH}_{*}\left(A^{\prime}, B, A^{\prime}\right)$ of $A^{\prime}$ are quasi-isomorphic.

Example 7.10. Assume $\operatorname{char}(k)=0$ and let $B$ be the group ring $k[G]$ for an arbitrary discrete abelian group $G$. Note that since $B$ is a cocommutative Hopf algebra, the functors $\mathrm{QCH}_{*}$ and $\mathrm{CH}_{*}$ are the same. Consider a $k[G]$-module field $A$ which is algebraically closed. In other words, $A$ is an algebraically closed field extension of $k$ such that $G$ acts by field automorphisms on $A$ and fixes the subfield $k$. We do not require $A^{G}=k$, but the reader may assume it for convenience. Let $\chi(G, A)$ denote the group of $A$-valued characters of $G$ and let $\operatorname{rep}_{A}^{G}$ be the category of finite dimensional $A$-vector spaces which admit an action of $G$. Since every such representation splits as a direct sum of irreducible representations and since every irreducible representation of $G$ is 1-dimensional over $A, \operatorname{rep}_{A}^{G}$ is generated by the subcategory of 1-dimensional representations. This subcategory is equivalent to a small category $\mathcal{A}$ whose set of objects is $\left\{A_{\delta} \mid \delta \in \chi(G, A)\right\}$ where we have $A_{\delta}=A$ as an $A$-vector space, and $A_{\delta}$ is a $G$-module via the character $\delta$. Any morphism of $A$ vector spaces between $A_{\delta}$ and $A_{\mu}$ for $\delta, \mu \in \chi(G, A)$ is given by an element of $A$, i.e. $\operatorname{Hom}_{\mathcal{A}}\left(A_{\delta}, A_{\mu}\right)=A$ as $A$-vector spaces. The composition of two morphisms $A_{\gamma} \stackrel{a}{\leftarrow}$ $A_{\mu} \stackrel{a^{\prime}}{\longleftarrow} A_{\delta}$ in $\mathcal{A}$ is defined to be the product $a a^{\prime}$ in $A$. The $G$-action on $\operatorname{Hom}_{\mathcal{A}}\left(A_{\delta}, A_{\mu}\right)$ is defined by

$$
(g \cdot a)(x):=g\left(a\left(g^{-1}(x)\right)\right)=\mu(g) \delta\left(g^{-1}\right) a x
$$

for any $g \in G, a \in \operatorname{Hom}_{\mathcal{A}}\left(A_{\delta}, A_{\mu}\right)$ and $x \in A_{\delta}$. If we define $\delta^{-1}(g)$ as the character given by the formula $\delta\left(g^{-1}\right)$ for any $g \in G$, then we see that $\operatorname{Hom}_{\mathcal{A}}\left(A_{\delta}, A_{\mu}\right) \cong A_{\mu \delta^{-1}}$ as $k[G]$-modules. Hence

$$
\begin{aligned}
\mathrm{QCH}_{n}\left(\mathcal{A}, k[G], \operatorname{Hom}_{\mathcal{A}}\right) & \cong \bigoplus_{\delta_{0}, \ldots, \delta_{n} \in \chi(G, A)} A_{\delta_{0} \delta_{1}^{-1}} \otimes \cdots \otimes A_{\delta_{n-1} \delta_{n}^{-1}} \otimes A_{\delta_{n} \delta_{0}^{-1}} \\
& \cong \bigoplus_{\mu_{0} \cdots \mu_{n}=\varepsilon} A_{\mu_{0}} \otimes \cdots \otimes A_{\mu_{n}},
\end{aligned}
$$

where $\varepsilon$ is the counit which is defined as $\varepsilon(g)=1$ for any $g \in G$. Note that since the product of all characters in each summand is the counit $\varepsilon$, the $G$-action on 
$\mathrm{QCH}_{*}\left(\mathcal{A}, k[G], \operatorname{Hom}_{\mathcal{A}}\right)$ is trivial. For two simplicial $k$-modules $X_{\bullet}$ and $Y_{\bullet}$, if we use $\operatorname{diag}\left(X_{\bullet} \otimes Y_{\bullet}\right)$ to denote the diagonal simplicial $k$-module structure on the product bi-simplicial module $X_{\bullet} \otimes Y_{\bullet}$, then we have

$$
\begin{aligned}
\mathrm{QCH}_{\bullet}\left(\operatorname{rep}_{A}^{G}\right) & \simeq \mathrm{CH}_{\bullet}(A[\chi(G, A)])_{\langle\varepsilon\rangle} \\
& \cong \operatorname{diag}\left(\mathrm{CH}_{\bullet}(A) \otimes \mathrm{CH}_{\bullet}(k[\chi(G, A)])_{\langle\varepsilon\rangle}\right)
\end{aligned}
$$

as simplicial $k[G]$-modules. Here we use $\mathrm{CH}_{\bullet}(k[\chi(G, A)])\langle\varepsilon\rangle$ to denote the simplicial submodule of the Hochschild complex $\mathrm{CH}_{\bullet}(k[\chi(G, A)])$ which is generated by elements of the form $\left(\delta_{0} \otimes \cdots \otimes \delta_{n}\right) \in \mathrm{CH}_{n}(k[\chi(G, A)])$ with the property that $\delta_{0} \cdots \delta_{n}=\varepsilon$ for $n \geqslant 0$. By using the Eilenberg-Zilber theorem and [15, Section 7.4], we conclude

$$
H H_{*}^{\mathrm{Hopf}}\left(\operatorname{rep}_{A}^{G}\right) \cong H H_{*}(A \mid k) \otimes H_{*}(\chi(G, A))
$$

as graded $k$-modules, where $H H_{*}(A \mid k)$ denotes the Hochschild homology of $A$ viewed as a $k$-algebra and $H_{*}(\chi(G, A))$ denotes the group homology of $\chi(G, A)$ with coefficients in $k$.

\section{Twisted equivariant bifunctors as coefficients}

In this section we assume $B$ is a Hopf algebra with an invertible antipode.

Definition 8.1. An arbitrary left-left $B$-module/comodule $M$ is called a YetterDrinfeld module if one has

$$
(b m)_{(-1)} \otimes(b m)_{(0)}=b_{(1)} m_{(-1)} S\left(b_{(3)}\right) \otimes b_{(2)} m_{(0)}
$$

for any $b \in B$ and $m \in M$, where we use Sweedler's notation for the coproduct on $H$ and for the $H$-coaction on $M$.

Definition 8.2. Assume $M$ is a Yetter-Drinfeld module over $B$. Let $\mathcal{C}$ be an arbitrary $B$-category and $\mathcal{H}$ be an arbitrary $B$-equivariant bifunctor on $\mathcal{C}$ with values in $B$-Mod. We define a new bifunctor $M \ltimes \mathcal{H}$ by letting $M \ltimes \mathcal{H}(X, Y):=$ $M \otimes \mathcal{H}(X, Y)$ on the objects for any $X, Y \in O b(\mathcal{C})$. Now we let

$$
b(m \otimes h)=b_{(1)}(m) \otimes b_{(2)}(h)
$$

for any $m \otimes h$ in $\mathcal{H}(X, Y)$. We define the bifunctor $M \ltimes \mathcal{H}$ on morphisms as follows: notice that such functors are defined by structure morphisms given in (6.1). Then we let

$$
(m \otimes h)(Y \stackrel{\beta}{\leftarrow} Z):=m \otimes \mathcal{H}\left(i d_{X}, \beta\right)(h)
$$

and

$$
(W \stackrel{\alpha}{\leftarrow} X)(m \otimes h):=m_{(0)} \otimes \mathcal{H}\left(S^{-1}\left(m_{(-1)}\right)(\alpha), i d_{Y}\right)(h)
$$

for any $m \otimes h$ in $M \ltimes \mathcal{H}(X, Y), \alpha \in \operatorname{Hom}_{\mathcal{C}}(W, X)$ and $\beta \in \operatorname{Hom}_{\mathcal{C}}(Y, Z)$. For simplicity we will denote $\mathcal{H}(\alpha, \beta)(h)$ by $\alpha h \beta$ for any $h \in \mathcal{H}(X, Y), \alpha \in \operatorname{Hom}_{\mathcal{C}}(W, X)$ and $\beta \in \operatorname{Hom}_{\mathcal{C}}(Y, Z)$.

Lemma 8.3. Let $B, M, \mathcal{C}$ and $\mathcal{H}$ be as in Definition 8.2. Then $M \ltimes \mathcal{H}$ is also a $B$-equivariant bifunctor on $\mathcal{C}$ with values in $B$-Mod. 
Proof. For $b \in B$ and $m \otimes h$ in $M \ltimes \mathcal{H}(X, Y), \alpha \in \operatorname{Hom}_{\mathcal{C}}(W, X)$, and finally $\beta \in$ $\operatorname{Hom}_{\mathcal{C}}(Y, Z)$, we consider

$$
\begin{aligned}
b((m \otimes h) \beta) & =b(m \otimes h \beta) \\
& =b_{(1)}(m) \otimes b_{(2)}(h \beta) \\
& =b_{(1)}(m) \otimes b_{(2)}(h)\left(b_{(3)} \beta\right) \\
& =b_{(1)}(m \otimes h)\left(b_{(2)} \beta\right)
\end{aligned}
$$

and

$$
\begin{aligned}
b(\alpha(m \otimes h)) & =b\left(m_{(0)} \otimes\left(S^{-1}\left(m_{(-1)}\right) \alpha\right)(h)\right) \\
& =b_{(1)}\left(m_{(0)}\right) \otimes\left(b_{(2)} S^{-1}\left(m_{(-1)}\right) \alpha\right) b_{(3)}(h) \\
& =b_{(2)(2)} m_{(0)} \otimes\left(b_{(2)(3)} S^{-1}\left(m_{(-1)}\right) S^{-1}\left(b_{(2)(1)}\right) b_{(1)} \alpha\right) b_{(3)}(h) \\
& =\left(b_{(1)} \alpha\right)\left(b_{(2)} m \otimes b_{(3)} h\right) \\
& =\left(b_{(1)} \alpha\right) b_{(2)}(m \otimes h),
\end{aligned}
$$

which implies $M \ltimes \mathcal{H}$ is a $B$-equivariant bifunctor on $\mathcal{C}$ with values in $B$-Mod whenever $\mathcal{H}$ is a bifunctor on $\mathcal{C}$ with values in $B$-Mod and $M$ is a Yetter-Drinfeld module on $B$.

For a $B$-module algebra $A$ and a Yetter-Drinfeld module $M$, we now define a new differential graded $B$-module $\mathrm{QCH}_{*}(A, B ; M)$ as $\mathrm{QCH}_{*}\left(*_{B}^{A}, B, M \ltimes \operatorname{Hom}_{A}(\cdot, \cdot)\right)$ and obtain twisted version of the Morita equivalence.

Theorem 8.4 (Morita invariance of twisted Hopf-Hochschild (co)homology). Let $B$ be a Hopf algebra and $M$ be a Yetter-Drinfeld module over $B$. Assume also that $A$ and $A^{\prime}$ are two $B$-module algebras. If the category of finitely generated $B$-equivariant representations of $A$ and $A^{\prime}$ are $B$-equivariantly equivalent, then the twisted Hopf-Hochschild complexes ${ }_{B} \mathrm{QCH}_{*}(A, B ; M)$ and ${ }_{B} \mathrm{QCH}_{*}\left(A^{\prime}, B ; M\right)$ are quasi-isomorphic.

\section{References}

[1] R. Akbarpour and M. Khalkhali, Equivariant cyclic cohomology of $\mathcal{H}$-algebras, K-Theory 29 (2003), 231-252.

[2] M. F. Atiyah and G. B. Segal, Equivariant $K$-theory and completion, $J$. Differential Geometry 3 (1969), 1-18.

[3] S. Baaj and G. Skandalis, $C^{*}$-algèbres de Hopf et théorie de Kasparov équivariante, K-Theory 2 (1989), 683-721.

[4] J. Block, E. Getzler, and J. D. S. Jones, The cyclic homology of crossed product algebras. II. Topological algebras, J. Reine Angew. Math. 466 (1995), 19-25.

[5] J.-L. Brylinski, Cyclic homology and equivariant theories, Ann. Inst. Fourier (Grenoble) 37 (1987), 15-28.

[6] C. Cibils and A. Solotar, Galois coverings, Morita equivalence and smash extensions of categories over a field, Documenta Math. 11 (2005), 143-159. 
[7] A. Connes and H. Moscovici, Hopf algebras, cyclic cohomology and the transverse index theorem, Comm. Math. Phys. 1998, 199-246.

[8] E. Getzler and J. D. S. Jones, The cyclic homology of crossed product algebras, J. Reine Angew. Math. 445 (1993), 161-174.

[9] P. Green, Equivariant K-Theory and Crossed Product $C^{*}$-Algebras, No. 38, pp. 337-338, Amer. Math. Soc., Providence, RI, 1982.

[10] P. M. Hajac, M. Khalkhali, B. Rangipour, and Y. Sommerhäuser, Hopf-cyclic homology and cohomology with coefficients, C. R. Math. Acad. Sci. Paris 338 (2004), 667-672.

[11] P. Julg, $K$-théorie équivarante et produits croisés, C. R. Math. Acad. Sci. Paris 292 (1981), 629-632.

[12] G. G. Kasparov, Equivariant $K K$-theory and the Novikov conjecture, Invent. Math. 91 (1988), 147-201.

[13] A. Kaygun, Bialgebra cyclic homology with coefficients, K-Theory 34 (2005), 151-194.

[14] A. Kaygun and M. Khalkhali, Bivariant Hopf cyclic cohomology, preprint; arXiv:math.KT/0606341.

[15] J.-L. Loday, Cyclic homology, Vol. 301 of Die Grundlehren der Mathematischen Wissenschaften, Springer-Verlag, Berlin, 1998.

[16] J. P. May, Simplicial Objects in Algebraic Topology, University of Chicago Press, Chicago, 1992.

[17] R. McCarthy, The cyclic homology of an exact category, J. Pure Appl. Algebra 93 (1994), 251-296.

[18] S. Neshveyev and L. Tuset, Hopf algebra equivariant cyclic cohomology, $K$-theory and index formulas, $K$-Theory 31 (2004), 357-378.

[19] T. Pirashvili and B. Richter, Hochschild and cyclic homology via functor homology, K-Theory 25 (2002), 39-49.

[20] M. Wodzicki, Excision in cyclic homology and in rational algebraic $K$-theory, Ann. of Math. 129 (1989), 591-639.

\author{
Atabey Kaygun kaygun@math.ohio-state.edu \\ Department of Mathematics \\ The Ohio State University \\ Columbus, Ohio 43210 \\ USA
}

This article is available at http://intlpress.com/HHA/v9/n2/a17 